\title{
A Joint-Channel Diagonalization for Multiuser MIMO Antenna Systems
}

\author{
Kai-Kit Wong, Member, IEEE, Ross D. Murch, Senior Member, IEEE, and Khaled Ben Letaief, Fellow, IEEE
}

\begin{abstract}
In this paper, we address the problem of improving the performance of multiuser space-division multiplexing (SDM) systems where multiple independent signal streams can be transmitted in the same frequency and time slot. The problem is important in multiuser multiple-input multiple-output systems where communication from one base station to many mobile stations can occur simultaneously. Our objective is to devise a multiuser linear space-time precoder for simultaneous channel diagonalization of the multiuser channels enabling SDM. Our new approach is based on diagonalizing the multiuser channel matrices and we use a variation of successive Jacobi rotations. In addition to the diagonalization, our approach attempts to optimize the resultant channel gains for performance enhancement. Our method is valid for both frequency-flat and frequency-selective fading channels but we assume that the base station knows all the channels and that they are quasi-stationary.
\end{abstract}

Index Terms-Capacity, co-channel interference (CCI), diversity, joint-channel diagonalization (JCD), multiple-input multiple-output (MIMO), multiuser communications, smart antennas, space-division multiplexing (SDM), spectral efficiency.

\section{INTRODUCTION}

C O-CHANNEL interference (CCI), as a result of frequency reuse, and intersymbol interference (ISI) caused by multipath fading have long been viewed as the major obstacles that limit the performance of high-speed wireless communications. To circumvent the impairments, various techniques such as channel coding, equalization, and interference cancellation have been investigated. One of the most promising techniques is through the use of smart or adaptive antennas. Several smart antenna systems have been proposed and demonstrated at the base station (BS) of the wireless communication system (e.g., [1], [2]). In recent years, performance enhancement utilizing multiple-input multiple-output [(MIMO) or multiple-transmit antennas and multiple-receive antennas] systems has been proposed [3]-[8]. In these systems, smart antennas are operated jointly at both the transmitter and receiver.

In this paper, we address the problem of enhancing the performance of multiuser MIMO systems for transmission from one BS to many mobile stations (MS) (or point-to-mul-

Manuscript received December 21, 2001; revised June 25, 2002 and August 28, 2002; accepted September 8, 2002. The editor coordinating the review of this paper and approving it for publication is F.-C. Zheng. This work was supported in part by the Hong Kong Research Grant Council (HKUST6024/01E) and in part by the Hong Kong Telecom Institute of Information Technology.

K.-K. Wong is with the Department of Electrical and Electronic Engineering, The University of Hong Kong, Hong Kong (e-mail: kitwong@ieee.org).

R. D. Murch and K. B. Letaief are with the Center for Wireless Information Technology, Department of Electrical and Electronic Engineering, The Hong Kong University of Science and Technology, Hong Kong.

Digital Object Identifier 10.1109/TWC.2003.814347 tipoint) in both frequency-flat and selective fading channels. To this end, we consider a linear multiuser precoder, operating in space-time, at the BS, and conventional maximal ratio combining (MRC) at all the MS for reception. Our objective is to determine the multiuser precoder for joint multiuser channel diagonalization in a synchronous multiuser channel, subject to the constraint of fixed user transmit power and that the transmitter (or BS) knows all the channels. Our approach is based on diagonalizing the matrices and we use a variation of successive Jacobi rotations. By doing so, we only need to determine four antenna weights among all the users' antenna weights at each step and this greatly reduces the system complexity compared with other schemes.

In [3], space-time or frequency codes that allow space-division multiplexing (SDM) were proposed for increasing the system capacity in the context of single user communication (point-to-point transmission). It was also demonstrated that extraordinary capacity could be achieved with or without channel state information (CSI) at the transmitter. More recently in [4], the capacity and the array gain of a perfectly optimized MIMO antenna system was studied under the consideration of adaptive power allocation among the parallel channels. Later, in [5], a solution using MIMO antenna to the broader problem of performance optimization for multipath frequency-selective fading channels in the presence of interference was provided. However, point-to-multipoint downlink communication using an antenna system has not been well studied. In addition to exploiting multiple signaling spatial dimension, space diversity can also be employed for support of multiple users, transmitting in the same frequency band and time slot [6]-[8]. In [6], joint optimal beamforming and power control was studied in a multipoint downlink scenario. However, the study was limited to the case that single antennas are used at the MS terminals. In [7], Wong et al. considered a multicarrier MIMO system in the context of multiuser communications. An iterative approach based on the flat-fading weights solution in [5] was proposed.

Our work is different in that we look into the problem of diagonalizing multiuser downlink channels simultaneously so that CCI can be nullified while at the same time the resultant channel gains can be optimized for maximizing the overall system capacity. Provided that this joint diagonalization exists, the resulting multiuser MIMO system will be decomposed into parallel uncoupled channels and users' data can be transmitted in disjoint space. One motivation of decoupling the multiuser channels is that users can be treated independently and some advanced techniques such as adaptive bit and power allocation can be used to further improve the individual user's performance without affecting the performance of other users. 


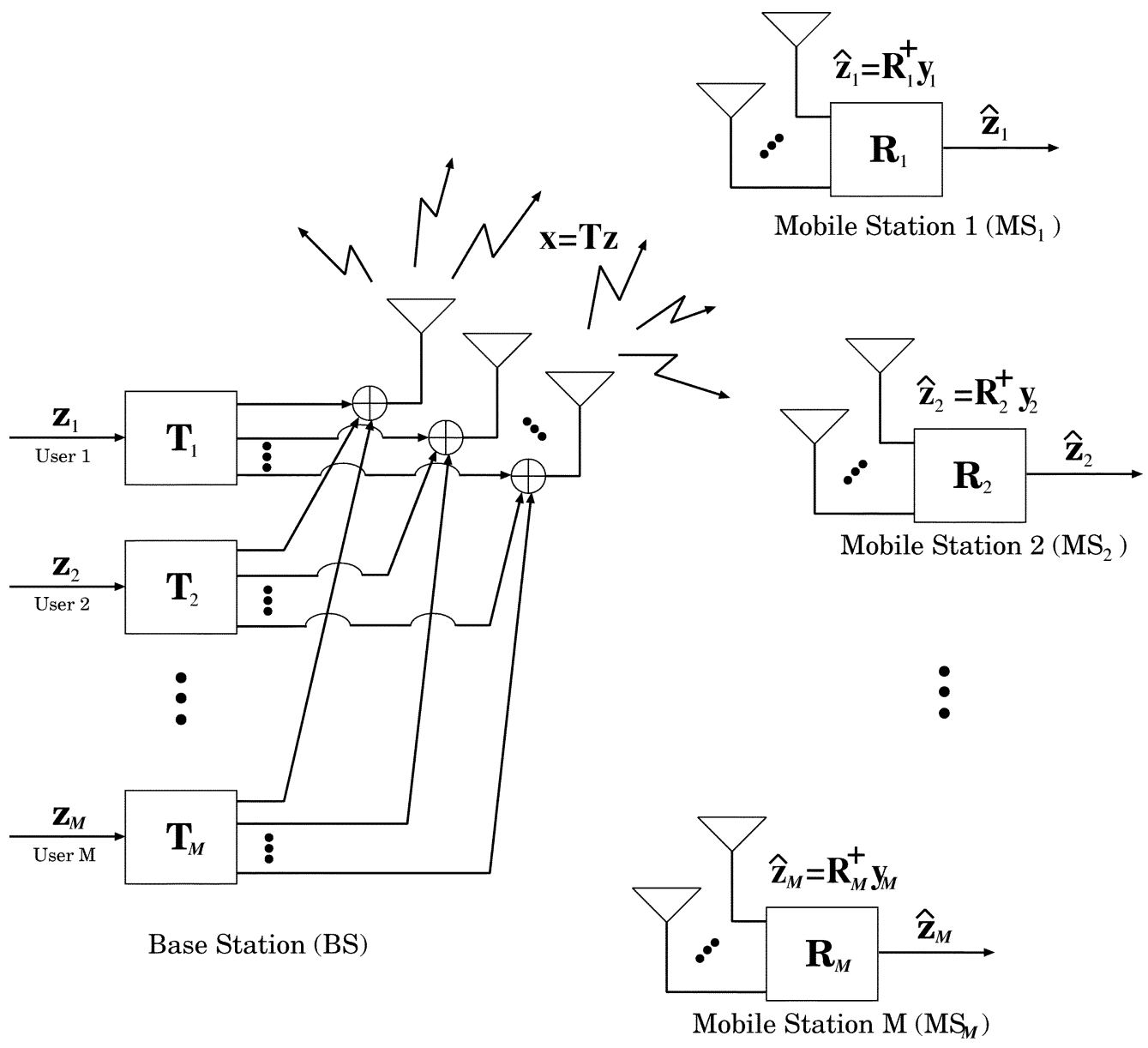

Fig. 1. System configuration of a multiuser MIMO antenna system.

Throughout this paper, we shall refer to this diagonalized system as a joint multiuser MIMO system (JMMS).

The assumption for this scheme is that the BS knows all the channels and the channel dynamics are quasi-stationary over a block of precoded bits as is typical of indoor low-mobility high-speed wireless communications. The proposed system is best implemented in time-division duplex mode as pilot symbols can be used for obtaining good estimates of CSI during uplink reception [9]-[11] and the estimates can be readily used for computing the antenna weights for downlink transmission. For indoor users, it is reasonable to have a small value of Doppler frequency, say $10 \mathrm{~Hz}$ (speed of $0.6 \mathrm{~m} / \mathrm{s}$ at $5 \mathrm{GHz}$ ), which corresponds to a coherence time of $40 \mathrm{~ms}$. Therefore, the channel would be relatively stationary as compared with the 2-ms frame duration of HiperLAN type 2 as an example.

The remainder of the paper is organized as follows. In Section II, we introduce the system model of a multiuser MIMO system. Section III proposes an iterative algorithm to determine the multiuser space-time precoder for a general multiuser MIMO system in multipath fading environments. In Section IV, simulation setup and results are presented. Finally, we have some concluding remarks in Section V.

\section{MultiUSER MIMO SYSTEM MODEL}

The system configuration of the multiuser MIMO antenna system is shown in Fig. 1 where one BS is transmitting to $M$
MS. For the MIMO system, $n_{T}$ antennas are located at the BS and $n_{R, m}$ antennas are located at the $m$ th MS. We first consider the link between the BS and a single user $m$. Data is transmitted in blocks of symbols of length $N$ and the number of spatial subchannels (spatial subchannels or spatial dimensions are the channels created from space, usually by distinguishing the signals received from different locations) per user is denoted by $K_{m}$. Therefore, the total number of symbols sent by the $m$ th user is $N_{m} \triangleq N K_{m}$ (or more generally $K_{m}=N_{m} / N$ can be a fractional number) and this is written in packet format: $\mathbf{z}_{m}=\left[z_{1,1}^{(m)} z_{2,1}^{(m)} \cdots z_{N, 1}^{(m)} z_{1,2}^{(m)} \cdots z_{N, 2}^{(m)} \cdots z_{N, K_{m}}^{(m)}\right]^{T}$, where $z_{n, k}^{(m)}$ is the $k$ th dimension of the $n$th symbol transmitted by the $m$ th user, and the superscript $T$ denotes the transpose operation. The packet is multiplied by a $N \times N_{m}$ transmission matrix

$$
\mathbf{T}_{k}^{(m)}=\left[\begin{array}{cccc}
t_{1,1}^{(m, k)} & t_{1,2}^{(m, k)} & \cdots & t_{1, N_{m}}^{(m, k)} \\
t_{2,1}^{(m, k)} & t_{2,2}^{(m, k)} & & \vdots \\
\vdots & & \ddots & \\
t_{N, 1}^{(m, k)} & \cdots & & t_{N, N_{m}}^{(m, k)}
\end{array}\right]
$$

to produce a packet $\mathbf{x}_{m, k}=\mathbf{T}_{k}^{(m)} \mathbf{z}_{m}$ which is transmitted by the $k$ th BS antenna to the $m$ th mobile in a block of length $N$.

The channel between the $k$ th BS antenna and $\ell$ th MS antenna is assumed quasi-stationary and can be considered as time-in- 
variant over a packet, so that it can be characterized by a Toeplitz matrix [5]

$$
\mathbf{H}_{\ell, k}^{(m)}=\left[\begin{array}{cccccc}
h_{0}^{(m, \ell, k)} & 0 & 0 & \ldots & & 0 \\
h_{1}^{(m, \ell, k)} & h_{0}^{(m, \ell, k)} & 0 & \ldots & & 0 \\
\vdots & \ddots & \ddots & \ddots & & \vdots \\
h_{\nu}^{(m, \ell, k)} & & \ddots & h_{0}^{(m, \ell, k)} & & \\
0 & \ddots & & & \ddots & 0 \\
\vdots & & & & \ddots & h_{0}^{(m, \ell, k)} \\
0 & & & & & h_{1}^{(m, \ell, k)} \\
\vdots & & & \ddots & \ddots & \vdots \\
0 & \ldots & & & 0 & h_{\nu}^{(m, \ell, k)}
\end{array}\right]
$$

where the maximum delay is assumed to last for $\nu$ samples and the discrete-time channel gains are defined by a multiray model, so the dimensions of $\mathbf{H}_{\ell, k}^{(m)}$ are $(N+\nu) \times N$.

At the MS, the received packet at the $\ell$ th antenna is given by $\mathbf{y}_{m, \ell}=\sum_{k=1}^{n_{T}} \mathbf{H}_{\ell, k}^{(m)} \mathbf{x}_{m, k}$ and is weighted in space and time by a matrix $\mathbf{R}_{\ell}^{(m)^{\dagger}}$, where

$$
\mathbf{R}_{\ell}^{(m)}=\left[\begin{array}{cccc}
r_{1,1}^{(m, \ell)} & r_{1,2}^{(m, \ell)} & \cdots & r_{1, N_{m}}^{(m, \ell)} \\
r_{2,1}^{(m, \ell)} & r_{2,2}^{(m, \ell)} & & \vdots \\
\vdots & & \ddots & \\
r_{N+\nu, 1}^{(m, \ell)} & \cdots & & r_{N+\nu, N_{m}}^{(m, \ell)}
\end{array}\right]
$$

and the superscript $\dagger$ denotes the conjugate transpose operation, to produce an estimate $\hat{\mathbf{z}}_{m}$ of the original packets. Writing the packet transmitted from all antennas as $\mathbf{x}_{m}=\left[\mathbf{x}_{m, 1} ; \mathbf{x}_{m, 2} ; \ldots ; \mathbf{x}_{m, n_{T}}\right]^{T}$ and the received data by all antennas as $\mathbf{y}_{m}=\left[\mathbf{y}_{m, 1} ; \mathbf{y}_{m, 2} ; \ldots ; \mathbf{y}_{m, n_{R, m}}\right]^{T}$, we can write the received signal of the entire MIMO system as

$$
\mathbf{y}_{m}=\mathbf{H}_{m} \mathbf{x}_{m}+\mathbf{n}_{m}
$$

where $\mathbf{n}_{m}$ is the noise vector that is assumed to be additive white Gaussian noise (AWGN) with power $\sigma_{n}^{2}$. Likewise, $\mathbf{H}_{m}$ is given by

$$
\mathbf{H}_{m}=\left[\begin{array}{cccc}
\mathbf{H}_{1,1}^{(m)} & \mathbf{H}_{1,2}^{(m)} & \cdots & \mathbf{H}_{1, n_{T}}^{(m)} \\
\mathbf{H}_{2,1}^{(m)} & \mathbf{H}_{2,2}^{(m)} & \cdots & \mathbf{H}_{2, n_{T}}^{(m)} \\
\vdots & & \ddots & \vdots \\
\mathbf{H}_{n_{R, m}, 1}^{(m)} & \cdots & & \mathbf{H}_{n_{R}, m}^{(m)}, n_{T}
\end{array}\right]
$$

where $\mathbf{H}_{\ell, k}^{(m)}$ is defined in (2). The estimate $\hat{\mathbf{z}}_{m}$ can then be written as

$$
\hat{\mathbf{z}}_{m}=\mathbf{R}_{m}^{\dagger} \mathbf{H}_{m} \mathbf{T}_{m} \mathbf{z}_{m}+\mathbf{R}_{m}^{\dagger} \mathbf{n}_{m}
$$

where $\mathbf{R}_{m} \triangleq\left[\mathbf{R}_{1}^{(m)} ; \mathbf{R}_{2}^{(m)} ; \ldots ; \mathbf{R}_{n_{R, m}}^{(m)}\right] \in \mathbb{C}^{(N+\nu) n_{R, m} \times N_{m}}$ denotes the space-time weights operating on the received signals, and $\mathbf{T}_{m} \triangleq\left[\mathbf{T}_{1}^{(m)} ; \mathbf{T}_{2}^{(m)} ; \ldots ; \mathbf{T}_{n_{T}}^{(m)}\right] \in \mathbb{C}^{N n_{T} \times N_{m}}$ denotes the space-time weights operating on the transmitted packet.

By considering all $M$ users, we obtain an $M$-user MIMO system as

$$
\hat{\mathbf{z}}_{m}=\mathbf{R}_{m}^{\dagger}\left(\sum_{m^{\prime}=1}^{M} \mathbf{H}_{m} \mathbf{T}_{m^{\prime}} \mathbf{z}_{m^{\prime}}+\mathbf{n}_{m}\right) \quad \forall m
$$

where $\mathbf{z}_{m^{\prime}} \in \mathbb{C}^{N_{m^{\prime}}}$ denotes the symbols transmitted from the $m^{\prime}$ th user.

Note that there are $N_{m}$ symbols transmitted in $N$ symbol durations by the $m$ th user, and it is known [3] that the number of spatial dimensions should be bounded by

$$
K_{m} \leq \frac{\operatorname{rank}\left\{\mathbf{H}_{m}\right\}}{N} \leq \min \left\{\left(1+\frac{\nu}{N}\right) n_{R, m}, n_{T}\right\} \quad \forall m .
$$

In addition, the above formulation assumes that $\mathbf{H}_{m}$ are uncorrelated with themselves and $\mathbf{z}_{m}$.

We also find it useful to define the multiuser transmit weight matrix

$$
\mathbf{T}=\left[\begin{array}{llll}
\mathbf{T}_{1} & \mathbf{T}_{2} & \cdots & \mathbf{T}_{M}
\end{array}\right]
$$

but note that a similar definition for a multiuser receive weight matrix is generally not possible as the number of antennas at each of the mobiles might be different.

\section{Multiuser Channel Diagonalization}

Our objective is to obtain a multiuser channel diagonalization that optimizes the performance of the multiuser MIMO system in the downlink. We do this by optimizing the transmit and receive antenna weights such that

$$
\left(\mathbf{T}, \mathbf{R}_{1}, \ldots, \mathbf{R}_{M}\right)_{\text {opt }}=\arg \max _{\mathbf{T}, \mathbf{R}_{1}, \ldots, \mathbf{R}_{M}} \operatorname{trace}\left\{\boldsymbol{\Lambda} \mathbf{\Lambda}^{\dagger}\right\}
$$

where trace $\{\cdot\}$ denotes the trace of the input matrix, and

$$
\boldsymbol{\Lambda} \triangleq\left[\begin{array}{ccc}
\boldsymbol{\Lambda}_{1} & \mathbf{0} & \cdots \\
\mathbf{0} & \boldsymbol{\Lambda}_{2} & \\
\vdots & & \ddots
\end{array}\right]
$$

in which $\boldsymbol{\Lambda}_{m}$ is defined by

$$
\mathbf{R}_{m}^{\dagger} \mathbf{H}_{m} \mathbf{T}=[\mathbf{0}_{1} \cdots \mathbf{0}_{m-1} \underbrace{\boldsymbol{\Lambda}_{m}}_{\begin{array}{c}
\text { mth } \\
\text { sub-block } \\
\text { matrix }
\end{array}} \mathbf{0}_{m+1} \cdots \mathbf{0}_{M}] \quad \forall m
$$

and

$$
\boldsymbol{\Lambda}_{m}=\operatorname{diag}\left(\lambda_{1}^{(m)}, \lambda_{2}^{(m)}, \ldots, \lambda_{N_{m}}^{(m)}\right)
$$

is of dimension $N_{m} \times N_{m}$. Likewise, $\mathbf{0}_{m^{\prime}}$ is the $m^{\prime}$ th subblock zero matrix of dimension $N_{m} \times N_{m^{\prime}}$. From definition (12), each subblock matrix corresponds to the signals transmitted for each user to mobile location $m$ and, hence, by making all of them 

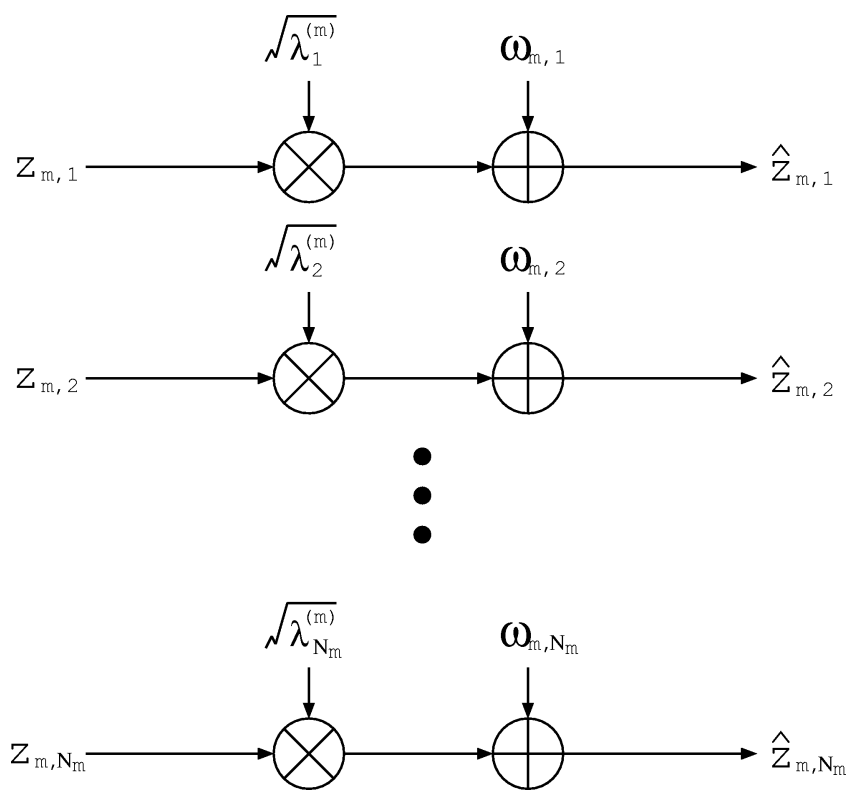

Fig. 2. Equivalent channel model of the $m$ th user where $w_{m, n}$ is white Gaussian noise with zero mean and $\sigma_{n}^{2}$ variance.

zero except for the $m$ th user, the CCI can be completely eliminated. The motivation for performing this diagonalization, if possible, is that the overall system becomes equivalent to uncoupled parallel channels for the mobiles (see Fig. 2) with their gains maximized so that

$$
\hat{\mathbf{z}}_{m}=\boldsymbol{\Lambda}_{m} \mathbf{z}_{m}+\mathbf{n}_{m}^{\prime}
$$

where $\mathbf{n}_{m}^{\prime}$ is another zero-mean complex AWGN noise vector with power $\sigma_{n}^{2}$, assuming the column vector of $\mathbf{R}_{m}$ is unit norm. The multiuser system capacity is then a simple extension of channel capacity for single-input single-output channels with memory [12] and can be expressed as

$$
\bar{C}=\frac{1}{N} \sum_{m=1}^{M} \log _{2} \operatorname{det}\left(\frac{\sigma_{z}^{2}\left|\boldsymbol{\Lambda}_{m}\right|}{\sigma_{n}^{2}}+\mathbf{I}\right)
$$

where $\operatorname{det}(\cdot)$ represents the determinant of the input matrix, $|\cdot|$ takes the modulus of every entry of the input matrix, and $\sigma_{z}^{2}=$ $\mathrm{E}\left[\left|z_{n, k}^{(m)}\right|^{2}\right]$.

We perform the optimization (12) and (10) under the following constraints. First, the transmit power at the BS is fixed at all time samples and invariant from sample to sample. This is done by constraining the radiated power of each transmit weight vector to be $P_{T}$. Therefore, adaptive modulation or "water-pouring" solution of adaptive power and information rate will not be considered in the analysis. (However, our results can be easily extended to the variable power case. This imposed constraint is in fact not necessary, but it simplifies the formulation.) Additionally, the rank requirement $\operatorname{rank}\left\{\mathbf{T}_{m}\right\}=N_{m}$ is imposed and assumed to be fixed during the optimization to ensure that all the transmitted symbols are received.
To begin, we first match the receiver to the channel to give $\mathbf{R}_{m}=\mathbf{H}_{m} \mathbf{T}_{m}$. This is essentially an MRC receiver and will be the optimum receiver in our configuration because the joint diagonalization we are using will nullify the interference terms. After doing so, it should be noted that $\boldsymbol{\Lambda}_{m}$ is now positive definite and would have nonnegative entries. Next, note that (12) can be rewritten as

$$
\begin{aligned}
\mathbf{T}^{\dagger} \mathbf{H}_{m}^{\dagger} \mathbf{H}_{m} \mathbf{T} & {\left[\begin{array}{ccccccc}
\times & \cdots & \times & \mathbf{0}_{1} & \times & \cdots & \times \\
\vdots & \ddots & & \vdots & \vdots & \ddots & \vdots \\
\times & \cdots & \times & \mathbf{0}_{m-1} & \times & \cdots & \\
\mathbf{0}_{1} & \cdots & \mathbf{0}_{m-1} & \boldsymbol{\Lambda}_{m} & \mathbf{0}_{m+1} & \cdots & \mathbf{0}_{M} \\
\times & \cdots & & \mathbf{0}_{m+1} & \times & \cdots & \\
\vdots & \ddots & & \vdots & \vdots & \ddots & \\
& & \times & \mathbf{0}_{M} & & & \times
\end{array}\right] \forall m }
\end{aligned}
$$

in which the symbol " $x$ " is an arbitrary matrix of appropriate size. In (16), the $\left(m_{1}, m_{2}\right)$ th subblock matrix $\mathbf{T}_{m_{1}}^{\dagger} \mathbf{H}_{m}^{\dagger} \mathbf{H}_{m} \mathbf{T}_{m_{2}}$ corresponds to the $m_{2}$ th user's signal components $\mathbf{T}_{m_{2}}$ received by the weight matrix $\mathbf{H}_{m} \mathbf{T}_{m_{1}}$ at the $m$ th mobile location $\mathbf{H}_{m}$. Obviously, at the $m$ th mobile location, the receive weight matrix is $\mathbf{H}_{m} \mathbf{T}_{m}$ and only the $m$ th user signals are to be detected. Therefore, the $\left(m_{1}, m_{2}\right)$ th subblock matrix with $m_{1} \neq m$ is not of our interest and is indicated by the symbol " $\times$." Our optimization (10) is then to find $\mathbf{T}$ so that

$$
\mathbf{T}_{\mathrm{opt}}=\arg \max _{\mathbf{T}} \operatorname{trace}\left\{\boldsymbol{\Lambda} \boldsymbol{\Lambda}^{\dagger}\right\}
$$

\section{A. Multiuser Optimization}

Performing our joint-channel diagonalization (JCD) (16) involves nullification of many dependent co-channel signals passing through different channels, and this makes a closed-form solution for the antenna weights extremely difficult to obtain. We have, therefore, resorted to a sequence of similarity updates that was inspired by the work of successive Jacobi rotations [14]. By doing so, the optimization can be reduced to a diagonalization problem of, at most, two channels, and this is more easily solved.

The sequence of similarity updates we invoke can be written as $\mathbf{A}_{m} \leftarrow \mathbf{Q}^{\dagger} \mathbf{A}_{m} \mathbf{Q} \forall m$ with initialization $\mathbf{A}_{m}=\mathbf{H}_{m}^{\dagger} \mathbf{H}_{m}$. Each new $\mathbf{A}_{m}$ can be thought of as an updated channel matrix for mobile $m$, which tends to more nearly satisfy our desired diagonalization (16) than its predecessor. At the first step, the transmit weight matrix is initialized as an identity matrix, $\mathbf{T}=$ $\mathbf{I}_{r}$. (In general, $\mathbf{T}$ is rectangular and, hence, $\mathbf{I}_{r}$ is rectangular.) After an adequate number of updates, (16) is eventually obtained. We can then use this to find $\mathbf{T}=\mathbf{I}_{r} \mathbf{Q}^{(1)} \mathbf{Q}^{(2)} \cdots \mathbf{Q}^{(U)}$, where $\mathbf{Q}^{(u)}$ is the updating matrix at the $u$ th step and $U$ is the total number of updates. The tools for doing this are transformations of the form (a variation of successive Jacobi rotations 
[14])

$$
\mathbf{Q}^{(u)}=\mathbf{Q}_{i, j}=\left[\begin{array}{ccccccc}
1 & \cdots & 0 & \cdots & 0 & \cdots & 0 \\
\vdots & \ddots & \vdots & & \vdots & & \vdots \\
0 & \cdots & p & \cdots & q & \cdots & 0 \\
\vdots & & \vdots & \ddots & \vdots & & \vdots \\
0 & \cdots & r & \cdots & s & \cdots & 0 \\
\vdots & & \vdots & & \vdots & \ddots & \vdots \\
0 & \cdots & 0 & \cdots & 0 & \cdots & 1
\end{array}\right] \quad j \text { th row }
$$

where $p$ is the $i$ th updating (space-time) element of the space-time weight vector for a certain user, say $m_{1}, q$ is the $i$ th updating element of the weight vector for another user $m_{2}, r$ is the $j$ th updating element of the weight vector for user $m_{1}$, and $s$ is the $j$ th updating element of the weight vector for the user $m_{2}$.

By letting $\mathbf{B}_{m}=\mathbf{Q}_{i, j}^{\dagger} \mathbf{A}_{m} \mathbf{Q}_{i, j}$, we can observe that the matrix $\mathbf{B}_{m}$ agrees with $\mathbf{A}_{m}$ except in rows and columns $i$ and $j$. As a result, we can reduce our optimization problem of each update by considering the following transformation:

$$
\mathbf{B}_{i, j}^{(m)}=\left[\begin{array}{ll}
p & q \\
r & s
\end{array}\right]^{\dagger} \mathbf{A}_{i, j}^{(m)}\left[\begin{array}{ll}
p & q \\
r & s
\end{array}\right] \quad \forall m
$$

where

$$
\mathbf{A}_{i, j}^{(m)} \triangleq\left[\begin{array}{cc}
a_{i, i}^{(m)} & a_{i, j}^{(m)} \\
a_{i, j}^{(m)^{*}} & a_{j, j}^{(m)}
\end{array}\right] \quad \text { and } \quad \mathbf{B}_{i, j}^{(m)} \triangleq\left[\begin{array}{cc}
b_{i, i}^{(m)} & b_{i, j}^{(m)} \\
b_{i, j}^{(m)^{*}} & b_{j, j}^{(m)}
\end{array}\right]
$$

in which $a_{i, j}^{(m)}$ and $b_{i, j}^{(m)}$ are, respectively, the $(i, j)$ th entries of $\mathbf{A}_{m}$ and $\mathbf{B}_{m}$. The $[p q ; r s]$ matrix is the two-element two-user weight matrix for transmitting signals for users $m_{1}$ and $m_{2}$. For each update, we only consider signals at mobile locations $m_{1}$ and $m_{2}$ and only the $i$ th and $j$ th space-time elements for users $m_{1}$ and $m_{2}$ are optimized. Signals at mobile locations $m \neq m_{1}, m_{2}$ are not of our interest. Following (16) and (17), the objective of each update is now to find the values of $p, q, r$, and $s$ so that

$$
\begin{aligned}
& \mathbf{B}_{i, j}^{\left(m_{1}\right)}=\left[\begin{array}{cc}
b_{i, i}^{\left(m_{1}\right)} & 0 \\
0 & \times
\end{array}\right] \\
& \mathbf{B}_{i, j}^{\left(m_{2}\right)}=\left[\begin{array}{cc}
\times & 0 \\
0 & b_{j, j}^{\left(m_{2}\right)}
\end{array}\right]
\end{aligned}
$$

(at the $m_{1}$ th mobile location)

(at the $m_{2}$ th mobile location)

and

$$
\max _{p, q, r, s}\left|b_{i, i}^{\left(m_{1}\right)}\right|^{2}+\left|b_{j, j}^{\left(m_{2}\right)}\right|^{2} .
$$

To perform this optimization, there are two possible situations.
- Single-User Case-When $m_{1}=m_{2}=m^{\star}$, the above optimization reduces to achieving

$$
\mathbf{B}_{i, j}^{\left(m^{\star}\right)}=\left[\begin{array}{cc}
b_{i, i}^{\left(m^{\star}\right)} & 0 \\
0 & b_{j, j}^{\left(m^{\star}\right)}
\end{array}\right]
$$

and the maximization of the sum $\left|b_{i, i}^{\left(m^{\star}\right)}\right|^{2}+\left|b_{j, j}^{\left(m^{\star}\right)}\right|^{2}$. This can be done readily by the eigenvalue decomposition (EVD) of $\mathbf{A}_{i, j}^{\left(m^{\star}\right)}$. As a consequence

$$
\left[\begin{array}{ll}
p & q \\
r & s
\end{array}\right]=\text { eigenmatrix of } \mathbf{A}_{i, j}^{\left(m^{\star}\right)} .
$$

- Two-User Case-When $m_{1} \neq m_{2}$, the optimal solution to jointly obtain (21)-(23) is unavailable. To deal with this, however, we use the solution to be presented in Section III-B [see (38)]. This solution can ensure the diagonalization of (21) and (22) while the individual gains $b_{i, i}^{\left(m_{1}\right)}$ and $b_{j, j}^{\left(m_{2}\right)}$ are maximized indirectly through the maximization of signal-to-interference ratio [defined later in (34) and (35)]. Using this solution, the values of $p, q, r$, and $s$ can be found by

$$
\left[\begin{array}{ll}
p & q \\
r & s
\end{array}\right]=\mathbf{W}_{i, j}^{\left(m_{2}\right)} \mathbf{F}
$$

where $\mathbf{W}_{i, j}^{\left(m_{2}\right)}$ is the whitening matrix such that $\mathbf{W}_{i, j}^{\left(m_{2}\right)^{\dagger}} \mathbf{A}_{i, j}^{\left(m_{2}\right)} \mathbf{W}_{i, j}^{\left(m_{2}\right)}=\mathbf{I}$, and $\mathbf{F}$ is the $2 \times 2$ matrix that contains the eigenvectors of $\mathbf{W}_{i, j}^{\left(m_{2}\right)^{\dagger}} \mathbf{A}_{i, j}^{\left(m_{1}\right)} \mathbf{W}_{i, j}^{\left(m_{2}\right)}$.

As a result, for each choice of $i<j$, we can find the transformation matrix $\mathbf{Q}_{i, j}$ that makes the composite channel approach (16) satisfied more closely. The details of how the iterations are carried out are summarized as follows.

1) Initialize $\mathbf{T}=\mathbf{I}_{r}$ and $\mathbf{A}_{m}=\mathbf{H}_{m}^{\dagger} \mathbf{H}_{m} \forall m$. Then, set $u=i=1$ and $j=2$.

2) Form an updating matrix $\mathbf{Q}^{(u)}=\mathbf{Q}_{i, j}$ based on (18). Identify the users $m_{1}$ and $m_{2}$ who correspond to, respectively, the $i$ th and $j$ th column vectors of $\mathbf{Q}_{i, j}$. If $m_{1}=m_{2}$, the values of $p, q, r$ and $s$ are found from (25), whereas (26) is used if $m_{1} \neq m_{2}$.

3) Compute

$$
\epsilon=|p-1|^{2}+|q|^{2}+|r|^{2}+|s-1|^{2} .
$$

If $\epsilon<$ Threshold ( $=10^{-12}$ typically), go to Step 5; otherwise, proceed to Step 4 . When having a nearly zero value of $\epsilon$, the updating matrix $[p q ; r s]$ or $\mathbf{Q}_{i, j}$ is close to an identity matrix, meaning that this updating is not necessary.

4) Update the effective channel matrices for all $m$ by

$$
\mathbf{A}_{m}=\mathbf{Q}_{i, j}^{\dagger} \mathbf{A}_{m} \mathbf{Q}_{i, j} .
$$

If $j<N n_{T}$, then update $j=j+1$; else update $i=i+1$ and $j=i+1$. But if $i=N n_{T}-1$ and $j=N n_{T}$, reset $i=1$ and $j=2$. Finally, update $u=u+1$. Then, go back to Step 2. 
5) The convergence is said to be achieved and the transmit weight matrix can be constructed by the multiplication of all the updating matrices, i.e.

$$
\mathbf{T}=\mathbf{I}_{r} \mathbf{Q}^{(1)} \mathbf{Q}^{(2)} \mathbf{Q}^{(3)} \cdots
$$

Normalization is then performed for all columns of $\mathbf{T}$ to satisfy the power constraint. The corresponding receive matrix for user $m$ can then be found by $\mathbf{R}_{m}=\mathbf{H}_{m} \mathbf{T}_{m}$.

We refer to the overall system as a JMMS or JCD. The proposed JMMS attempts to maximize (17) [and, hence, the overall system capacity (15)], and transmits multiuser signals in disjoint space-time subchannels. Although we cannot prove (the difficulty of having such a proof is that in general $\mathbf{T}$ does not have to be orthogonal) that JMMS achieves (16), numerical results we have carried out suggest that JMMS is a way to achieve (16) as long as the necessary conditions for the minimum number of transmit and receive antennas are satisfied (will be discussed in Section III-C).

Intuition about this scheme can be obtained by considering a special case of a two-user system with three BS antennas, three antennas per MS where MS 1 transmits two dimensions and MS 2 transmits one dimension (i.e., $K_{1}=2, K_{2}=1$ ) in flat-fading channels (so we can consider $N=1$ ). Following the above Steps 1-5, we will initialize $\mathbf{T}=\mathbf{I}$ and $\mathbf{A}_{1}=\mathbf{H}_{1}^{\dagger} \mathbf{H}_{1}$ and $\mathbf{A}_{2}=$ $\mathbf{H}_{2}^{\dagger} \mathbf{H}_{2}$. Then, the updating matrix $\mathbf{Q}_{1,2}$ is found using (25) and the matrices $\mathbf{Q}_{1,3}$ and $\mathbf{Q}_{2,3}$ are found using (26). Updating will continue until convergence and the joint transmit weight matrix is given by

$$
\mathbf{T}=\mathbf{Q}_{1,2} \mathbf{Q}_{1,3} \mathbf{Q}_{2,3} \mathbf{Q}_{1,2}^{\prime} \mathbf{Q}_{1,3}^{\prime} \cdots
$$

where $\mathbf{Q}_{i, j}^{\prime}$ denotes the updating matrix in the second round iteration.

After going through the above iteration, multiuser diagonalization could be obtained so that

$$
\mathbf{T}^{\dagger} \mathbf{H}_{1}^{\dagger} \mathbf{H}_{1} \mathbf{T}=\left[\begin{array}{ccc}
\lambda_{1}^{(1)} & 0 & 0 \\
0 & \lambda_{2}^{(1)} & 0 \\
0 & 0 & \times
\end{array}\right]
$$

and

$$
\mathbf{T}^{\dagger} \mathbf{H}_{2}^{\dagger} \mathbf{H}_{2} \mathbf{T}=\left[\begin{array}{ccc}
\times & \times & 0 \\
\times & \times & 0 \\
0 & 0 & \lambda_{1}^{(2)}
\end{array}\right]
$$

where $\times$ means the "do not care" and this corresponds to the unused subchannel gains. The corresponding system spectral efficiency is then given by

$$
\bar{C}=\log _{2}\left(1+\frac{\sigma_{z}^{2} \lambda_{1}^{(1)}}{\sigma_{n}^{2}}\right)\left(1+\frac{\sigma_{z}^{2} \lambda_{2}^{(1)}}{\sigma_{n}^{2}}\right)\left(1+\frac{\sigma_{z}^{2} \lambda_{1}^{(2)}}{\sigma_{n}^{2}}\right) .
$$

A detailed study of the complexity issue of the algorithm is beyond the scope of this paper. However, some ideas can be gained from this example. When computing the updating matrices, $\mathbf{Q}_{1,2}, \mathbf{Q}_{1,2}^{\prime}, \ldots$, the number of floating point operations (flops) is $O(8)$, while the other updating matrices require $O(16)$ flops. The number of iterations for convergence in general depends upon the sizes of the channel matrices and the number of users. In this example, on average, the number of iterations required is $16(U=16)$.

\section{B. Two-User Optimization}

A key part of the solution to the optimization (17) described in the above section is a linear space-time precoder for simultaneous channel diagonalization of a two-user system [used in (26)]. To perform this, we make use of a closed-form solution for a linear space-time precoder given in [8]. This solution has been shown to be effective, especially when the number of users or the number of antennas at MS is small. In the following, we shall show that the solution in [8], with a little modification, can be used to simultaneously diagonalize two users' channels.

Consider a special case of a two-user system $(M=2)$, with two antennas at the BS $\left(n_{T}=2\right)$, and both MS has two antennas $\left(n_{R, 1}=n_{R, 2}=2\right)$ and transmits in only one dimension per user link (i.e., $K_{1}=K_{2}=1$ ) in flat-fading channels. In a flat-fading radio environment, ISI is negligible, so that the same set of weights can be used for the entire packet (i.e., $N=1$ ) [5]. Denoting the transmit weight vectors for User 1 and 2, respectively, as $\mathbf{t}^{(1)}=[p r]^{T}$ and $\mathbf{t}^{(2)}=\left[\begin{array}{ll}q s\end{array}\right]^{T}$, performance enhancement is done through the following maximization:

$$
\left.\mathbf{t}^{(1)}\right|_{\mathrm{opt}}=\left[\begin{array}{c}
p \\
r
\end{array}\right]=\arg \max _{\mathbf{t}^{(1)}} \frac{\mathbf{t}^{(1)^{\dagger}} \mathbf{H}_{1}^{\dagger} \mathbf{H}_{1} \mathbf{t}^{(1)}}{\mathbf{t}^{(1)^{\dagger}}\left(\frac{P_{T} \sigma_{z}^{2} \mathbf{H}_{2}^{\dagger} \mathbf{H}_{2}}{\sigma_{n}^{2}}+\mathbf{I}\right) \mathbf{t}^{(1)}}
$$

and

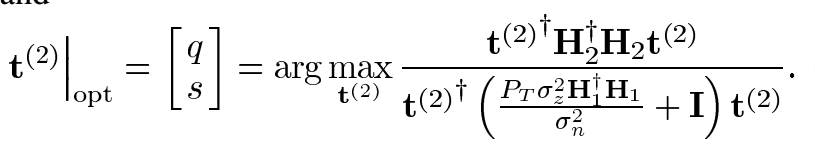

Here, instead of performing the above maximizations directly, as in [8], we focus on the case when the signal-to-noise ratio (SNR) is large (i.e., $P_{T} \sigma_{z}^{2} / \sigma_{n}^{2} \gg 1$ ). Therefore, the noise term (or the identity matrix in the denominator) could be ignored. To solve for the weights that maximize (34) and (35), we begin by writing the EVD of the channels as $\mathbf{H}_{1}^{\dagger} \mathbf{H}_{1}=\mathbf{V}_{1} \mathbf{D}_{1} \mathbf{V}_{1}^{\dagger}$ and $\mathbf{H}_{2}^{\dagger} \mathbf{H}_{2}=\mathbf{V}_{2} \mathbf{D}_{2} \mathbf{V}_{2}^{\dagger}$. Also, let $\mathbf{t}^{(1)}=\mu_{1} \mathbf{V}_{2} \mathbf{D}_{2}^{-(1 / 2)} \mathbf{e}_{1}$, where $\mu_{1}$ is a real constant that restricts the norm of $\mathbf{t}^{(1)}$ to be $\sqrt{P_{T}}$, and the superscript $-(1 / 2)$ denotes the inverse of the square root of a matrix. (When the matrix is singular or the channel $\mathbf{H}_{m}$ is rectangular, a Moore-Penrose pseudoinverse is used. A matrix $\mathbf{A}$ is said to be the square root of $\mathbf{B}$ if $\mathbf{B}=\mathbf{A} \mathbf{A}^{\dagger}$.) Then, the expression in (34) is maximized when $\mathbf{e}_{1}=\mathbf{e}_{\max }$, the eigenvector that corresponds to the largest eigenvalue of $\left(\mathbf{V}_{2} \mathbf{D}_{2}^{-(1 / 2)}\right)^{\dagger} \mathbf{H}_{1}^{\dagger} \mathbf{H}_{1}\left(\mathbf{V}_{2} \mathbf{D}_{2}^{-(1 / 2)}\right)$.

A similar solution for $\mathbf{t}^{(2)}$ can be found to maximize (35). However, we note that (35) can be thought of as a minimization problem. That is

$$
\max _{\mathbf{t}^{(2)}} \frac{1}{\left[\frac{\mathbf{t}^{(2) \dagger} \mathbf{H}_{1}^{\dagger} \mathbf{H}_{1} \mathbf{t}^{(2)}}{\mathbf{t}^{(2) \dagger} \mathbf{H}_{2}^{\dagger} \mathbf{H}_{2} \mathbf{t}^{(2)}}\right]} \equiv \min _{\mathbf{t}^{(2)}} \frac{\mathbf{t}^{(2)^{\dagger}} \mathbf{H}_{1}^{\dagger} \mathbf{H}_{1} \mathbf{t}^{(2)}}{\mathbf{t}^{(2)^{\dagger}} \mathbf{H}_{2}^{\dagger} \mathbf{H}_{2} \mathbf{t}^{(2)}} .
$$


As a result, the weight vector that maximizes (35) or equivalently minimizes (36) can be written as

$$
\mathbf{t}^{(2)}=\mu_{2} \mathbf{V}_{2} \mathbf{D}_{2}^{-(1 / 2)} \mathbf{e}_{\min }
$$

where $\mu_{2}$ is a real constant and $\mathbf{e}_{\min }$ is the eigenvector that corresponds to the least eigenvalue of $\left(\mathbf{V}_{2} \mathbf{D}_{2}^{-(1 / 2)}\right)^{\dagger} \mathbf{H}_{1}^{\dagger} \mathbf{H}_{1}\left(\mathbf{V}_{2} \mathbf{D}_{2}^{-(1 / 2)}\right)$. As a consequence, the solution for $\mathbf{t}^{(1)}$ and $\mathbf{t}^{(2)}$ that jointly performs (34) and (35) is given by

$$
\left[\mathbf{t}^{(1)} \mathbf{t}^{(2)}\right]=\left[\begin{array}{ll}
p & q \\
r & s
\end{array}\right]=\left[\begin{array}{cc}
\mu_{1} & 0 \\
0 & \mu_{2}
\end{array}\right] \mathbf{W}_{2} \mathbf{E}_{1}
$$

where $\mathbf{W}_{2}=\mathbf{V}_{2} \mathbf{D}_{2}^{-(1 / 2)}$, and $\mathbf{E}_{1}$ is the matrix whose columns are the eigenvectors of $\mathbf{W}_{2}^{\dagger} \mathbf{H}_{1}^{\dagger} \mathbf{H}_{1} \mathbf{W}_{2}$. Equation (38) is the solution we use in (26).

\section{Necessary Conditions for JCD}

We are interested in finding necessary conditions for (12) or (16) to exist. Given the set $\left\{K_{m}\right\}_{m=1}^{M}$, we find necessary conditions for the minimum required number of antennas at the BS $\left(n_{T}\right)$ and $\mathrm{MS}\left(\left\{n_{R, m}\right\}_{m=1}^{M}\right)$ for JCD.

Theorem 1-The Minimum Number of Transmit Antennas: The JCD (16) is possible only if $n_{T} \geq K_{1}+K_{2}+\cdots+K_{M}$.

Proof: From (12)

$$
\boldsymbol{\Lambda}=\left[\begin{array}{c}
\mathbf{R}_{1}^{\dagger} \mathbf{H}_{1} \\
\mathbf{R}_{2}^{\dagger} \mathbf{H}_{2} \\
\vdots \\
\mathbf{R}_{M}^{\dagger} \mathbf{H}_{M}
\end{array}\right] \mathbf{T} .
$$

Obviously, we want $\operatorname{rank}(\boldsymbol{\Lambda})=N_{1}+N_{2}+\cdots+N_{M}=$ $N\left(K_{1}+K_{2}+\cdots+K_{M}\right)$, where $N_{m}$ denotes the number of symbols transmitted in $N$ symbol durations by the $m$ th user. Therefore, from the rank property of matrices [for any two matrices $\mathbf{A}$ and $\mathbf{B}, \operatorname{rank}(\mathbf{A B}) \leq \min \{\operatorname{rank}(\mathbf{A}), \operatorname{rank}(\mathbf{B})\}]$ of matrices [13], we get

$$
\begin{array}{r}
N_{1}+N_{2}+\cdots+N_{M} \\
\leq \min \left\{\operatorname{rank}\left(\left[\begin{array}{c}
\mathbf{R}_{1}^{\dagger} \mathbf{H}_{1} \\
\mathbf{R}_{2}^{\dagger} \mathbf{H}_{2} \\
\vdots
\end{array}\right]\right), \operatorname{rank}(\mathbf{T})\right\} .
\end{array}
$$

Thus

$$
K_{1}+K_{2}+\cdots+K_{M} \leq n_{T} .
$$

In what follows, we assume that $n_{T} \geq K_{1}+K_{2}+\cdots+K_{M}$.

It is important to note that the number of transmit antennas is constrained by the number of spatial dimensions and not by the number of receive antennas. In fact, the total number of receive antennas may be much larger than the number of transmit antennas.

Theorem 2-The Minimum Number of Receive Antennas: The JCD (16) is possible only if $(N+\nu) n_{R, m} \geq N_{m}+1$. For flat-fading channels (i.e., $\nu=0$ or effectively $N=1$ ), we must have $n_{R, m} \geq K_{m}+1$.

Proof: Note from (16) that for MS $m$, there are at least $N_{m}+1$ orthogonal channels created after such diagonalization $-N_{m}$ desired channels and one interference channel (dedicated for all the "do not care" channels). Although we are not interested in the interference channels, we do need these to be completely orthogonal to the desired channels. As such

$$
\operatorname{rank}\left\{\mathbf{H}_{m} \mathbf{T}\right\} \geq N_{m}+1 \quad \forall m
$$

must be satisfied. Because of the assumption that $n_{T} \geq K_{1}+$ $\cdots+K_{M}$, we shall always consider the case when $N n_{T} \geq$ $N_{m}+1$. Accordingly, (42) implies that

$$
(N+\nu) n_{R, m} \geq N_{m}+1 .
$$

\section{RESULTS AND COMPARISONS}

The proposed JMMS system is investigated for a time-division multiple-access-based wireless communication system in flat Rayleigh-fading channels under the assumption of perfect CSI knowledge. To assess the system performance, we provide the average system spectral efficiency and average user bit-error probability results for various AWGN (SNR). The system spectral efficiency or capacity we use in this paper refers to the maximum achievable throughput of a given transmission algorithm. For each simulation, data packets consisting of 50 data symbols (i.e., $N=50$ ) are transmitted with more than 10000 independent channel realizations. To benchmark the results we obtain, we compare our results with an upper bound and also various alternative methods. These reference methods are summarized in the following.

\section{A. Benchmarks}

1) Performance Bound: The performance bound we use assumes no CCI is present and makes use of the singular value decomposition (SVD) for every user link (which is optimal in the absence of CCI). Also note that the bound does not involve $\mathrm{CCI}$ or assumes that $\mathrm{CCI}$ is completely eliminated. Hence, it is the upper bound for the true system capacity. It should also be emphasized that this performance bound is generally not achievable, unlike the Shannon capacity bound.

Using this approach, we write the transmit weight matrix for the $m$ th user as

and

$$
\mathbf{T}_{m}=\sqrt{P_{T}} \mathbf{V}_{m}
$$

$$
\mathbf{R}_{m}=\mathbf{U}_{m}
$$

where $\mathbf{V}_{m}\left(\mathbf{U}_{m}\right)$ is the matrix whose columns are the right (left) singular vectors which correspond to the $N_{m}$ largest singular values of $\mathbf{H}_{m}$, and the norm of each column vector of $\mathbf{T}_{m}$ is $\sqrt{P_{T}}$ (satisfying the power constraint).

2) ISBM Antenna System: In [5], an analytical formula for the MIMO antenna weights is derived in frequency-selective fading channels under CCI conditions. To deal with a multiuser 
system, we can make use of the solution for each user, and do it iteratively from user to user. This algorithm is referred to as iterative smart base and mobile (ISBM) antenna system.

Denoting $\mathbf{T}_{m}^{(i)}$ as the transmit weight matrix of the $m$ th user at the $i$ th iteration, the iterative algorithm can be formalized as follows.

- Step 1: Initialize $\mathbf{T}_{m}^{(0)}=\mathbf{I}$ for all $m$, and $i=0$.

- Step 2: For $i=1, \ldots, I$, let

$$
\mathbf{T}_{m}^{(i+1)}=\sqrt{P_{T}} \mathbf{F}_{m} \quad \forall m
$$

where $\mathbf{F}_{m}$ is the matrix that contains the eigenvectors that correspond to the $N_{m}$ largest eigenvalues of $\mathbf{H}_{m}^{\dagger} \boldsymbol{\Phi}_{m}^{(i)}{ }^{-1} \mathbf{H}_{m}$ where

$$
\boldsymbol{\Phi}_{m}^{(i)}=\mathbf{H}_{m}\left[\sigma_{z}^{2} \sum_{\substack{n=1 \\ n \neq m}}^{M} \mathbf{T}_{n}^{(i)} \mathbf{T}_{n}^{(i)^{\dagger}}\right] \mathbf{H}_{m}^{\dagger}+\sigma_{n}^{2} \mathbf{I} .
$$

- Step 3: $\mathbf{T}_{m}^{(I)}$ is the solution for the transmit weight matrices and the corresponding receive weight matrices are given by

$$
\mathbf{R}_{m}^{(I)}=\boldsymbol{\Phi}_{m}^{(I)}{ }^{-1} \mathbf{H}_{m} \mathbf{T}_{m}^{(I)}
$$

where $I$ is the total number of iterations.

In order to have fast convergence, a good choice for an initial guess of $\mathbf{T}_{m}^{(0)}$ is important. A logical guess is the smart base and mobile solution for frequency-selective fading channels in the absence of CCI [5]. Accordingly, throughout, the following initialization

$$
\mathbf{T}_{m}^{(0)}=\sqrt{P_{T}} \mathbf{E}_{m}
$$

is used where $\mathbf{E}_{m}$ is the matrix that contains the eigenvectors that correspond to the $N_{m}$ largest eigenvalues of $\mathbf{H}_{m}^{\dagger} \mathbf{H}_{m}$.

3) Maximum Transmit SINR: In [8], it was shown that the product SINR is lower bounded by

$$
\begin{aligned}
& \prod_{m=1}^{M} \prod_{k=1}^{K_{m}} \prod_{n=1}^{N} \gamma_{n, k}^{(m)} \\
& \geq \rho \prod_{m, k, n} \frac{\mathbf{t}_{n, k}^{(m)^{\dagger}} \mathbf{H}_{m}^{\dagger} \mathbf{H}_{m} \mathbf{t}_{n, k}^{(m)}}{\prod_{\substack{\tilde{m}=1 \\
\tilde{m} \neq m}}^{M}\left[\mathbf{t}_{n, k}^{(m) \dagger}\left(\frac{P_{T} \sigma_{z}^{2} \mathbf{H}_{\tilde{m}}^{\dagger} \mathbf{H}_{\tilde{m}}}{\sigma_{n}^{2}}+\mathbf{I}\right) \mathbf{t}_{n, k}^{(m)}\right]^{Q_{\tilde{m}}}}
\end{aligned}
$$

where $\gamma_{n, k}^{(m)}$ and $\mathbf{t}_{n, k}^{(m)}$ denote, respectively, the SINR and transmit weight vector of the $k$ th dimension of the $n$th symbol from the $m$ th user, $\rho$ is a constant, and

$$
Q_{\tilde{m}}= \begin{cases}K_{\tilde{m}} N, & \tilde{m} \neq m \\ K_{m} N-1, & \tilde{m}=m .\end{cases}
$$

It can be further shown that the lower bound can be maximized by

$$
\max _{\mathbf{t}_{n, k}^{(m)}} \frac{\mathbf{t}_{n, k}^{(m)^{\dagger}} \mathbf{H}_{m}^{\dagger} \mathbf{H}_{m} \mathbf{t}_{n, k}^{(m)}}{\mathbf{t}_{n, k}^{(m)}\left[\sum_{\substack{\tilde{m}=1 \\ \tilde{m} \neq m}}^{M} Q_{\tilde{m}}\left(\frac{P_{T} \sigma_{z}^{2} \mathbf{H}_{\tilde{m}}^{\dagger} \mathbf{H}_{\tilde{m}}}{\sigma_{n}^{2}}+\mathbf{I}\right)\right] \mathbf{t}_{n, k}^{(m)}}
$$

for all $m, n, k$. To find the weights that maximize (52), we begin by letting $\mathbf{t}_{n, k}^{(m)}=\mathbf{W}_{m} \mathbf{f}_{n, k}^{(m)}$ such that $\mathbf{W}_{m}$ satisfies

$$
\mathbf{W}_{m}^{\dagger}\left[\sum_{\substack{\tilde{\tilde{m}=1} \\ \tilde{m} \neq m}}^{M} Q_{\tilde{m}}\left(\frac{P_{T} \sigma_{z}^{2} \mathbf{H}_{\tilde{m}}^{\dagger} \mathbf{H}_{\tilde{m}}}{\sigma_{n}^{2}}+\mathbf{I}\right)\right] \mathbf{W}_{m}=\mathbf{I} .
$$

For a given $m, \mathbf{f}_{n, k}^{(m)}$ must all be distinct or orthogonal (by the rank constraint). Thus, (52) is maximized when $\mathbf{f}_{n, k}^{(m)}$ is the eigenvector that corresponds to the $((n-1) N+k)$ th largest eigenvalue of $\mathbf{W}_{m}^{\dagger} \mathbf{H}_{m}^{\dagger} \mathbf{H}_{m} \mathbf{W}_{m}$. In this paper, we shall refer to this system as maximum transmit SINR (MTXSINR).

4) Direct Transmission: A straightforward approach, which we refer to as a direct transmission (DTx) system, is considered. This approach partitions the transmit antennas and directly assigns different antennas to different users. As such, the transmit weights are

$$
\left[\begin{array}{llll}
\mathbf{T}_{1} & \mathbf{T}_{2} & \cdots & \mathbf{T}_{M}
\end{array}\right]=\mathbf{I}_{K}
$$

where $\mathbf{I}_{K}$ is a $K \times K$ identity matrix, and $K=\sum_{m=1}^{M} N_{m}$.

The advantage of DTx is that no prior knowledge of CSI is required at the transmitter, as compared with the other schemes presented in this section.

5) SVD-Minimum Mean-Square Error: For a single-user MIMO system, it is found [3] that the best way to transmit data into multiple spatial dimensions is through the use of SDM, by SVD of the channel matrix. In a multiuser system, we can use the method to distribute the data across space for increasing the capacity or spectral efficiency of the system. As a result, the transmit weights are found from (44). In addition, because of CCI, the receive weights need to be found from a traditional smart antenna algorithm for CCI suppression [17]. This system uses SVD for transmitting and minimum mean square error (MMSE) for reception. Thus, we refer to this system as a SVD-MMSE system.

6) Joint Approximate Diagonalization of Eigenmatrices: In [18], Cardoso and Souloumiac proposed an iterative approach for the joint approximate diagonalization of eigenmatrices (JADE). It suggests that for a set of $M$ complex hermitian matrices $\mathbf{G}_{m}$, it is possible to find a unitary matrix $\mathbf{U}$ that minimizes

$$
\sum_{m=1}^{M} \text { off }\left(\mathbf{U} \mathbf{G}_{m} \mathbf{U}^{\dagger}\right)
$$

where

$$
\operatorname{off}(\mathbf{A}) \triangleq \sum_{1 \leq k \neq \ell \leq N}\left|a_{k, \ell}\right|^{2}
$$


The idea is to maximize the desired or diagonal signals and minimize the undesired or off-diagonal signals. However, it should be noted that not all diagonal elements are used. Therefore, there will be some loss in the degree of freedom for adapting the weights. Using JADE on the matrices $\mathbf{H}_{m}^{\dagger} \mathbf{H}_{m}$, we obtain

$$
\left[\begin{array}{llll}
\mathbf{T}_{1} & \mathbf{T}_{2} & \cdots & \mathbf{T}_{M}
\end{array}\right]=\mathbf{U}
$$

for the weights operating on the transmitted packets while MMSE can be used as the weights operating on the received signals in minimizing the interuser and interchannel interference.

7) Multiuser MIMO Capacity: Since JMMS and the upper bound are based on diagonalization and uncoupled channels result, capacity is readily calculated (15). However, for the alternate methods (Sections IV-A2-IV-A6), where CCI is still present, multiuser capacity cannot be explicitly expressed because of the interference between co-channel signals. To allow performance comparisons in addition to bit-error rate (BER), we introduce an approximate capacity measure. Such a measure is based on the use of the Gaussian assumption which implies that the interference becomes Gaussian distributed when there are several users.

Suppose that we have a linear model similar to (4)

$$
\mathbf{y}=\mathbf{H x}+\mathbf{u}
$$

where $\mathbf{u}$ is the undesired signal vector which can be correlated noise or CCI. Assuming that $\mathbf{u}$ is Gaussian with covariance matrix $\mathrm{E}\left[\mathbf{u u}^{\dagger}\right]=\boldsymbol{\Phi}$, and is uncorrelated with $\mathbf{x}$. From [15], [16], we know that the capacity is of the form

$$
C=\log _{2} \frac{\operatorname{det}\left(\mathrm{E}\left[\mathbf{x} \mathbf{x}^{\dagger}\right]\right) \cdot \operatorname{det}\left(\mathrm{E}\left[\mathbf{y} \mathbf{y}^{\dagger}\right]\right)}{\operatorname{det}\left[\begin{array}{ll}
\mathrm{E}\left[\mathbf{x} \mathbf{x}^{\dagger}\right] & \mathrm{E}\left[\mathbf{x} \mathbf{y}^{\dagger}\right] \\
\mathrm{E}\left[\mathbf{y} \mathbf{x}^{\dagger}\right] & \mathrm{E}\left[\mathbf{y} \mathbf{y}^{\dagger}\right]
\end{array}\right]}
$$

After some manipulation, (59) can be simplified as

$$
C=\log _{2} \frac{\operatorname{det}\left(\mathbf{H E}\left[\mathbf{x} \mathbf{x}^{\dagger}\right] \mathbf{H}^{\dagger}+\mathbf{\Phi}\right)}{\operatorname{det} \boldsymbol{\Phi}} .
$$

The multiuser MIMO channel capacity is just a simple extension of (60). Using the system model defined in Section II, the total capacity per transmission of the $m$ th user link, $C_{m}$, can be found by

$$
C_{m}=\log _{2} \frac{\operatorname{det}\left(\mathbf{H}_{m} \mathrm{E}\left[\mathbf{x}_{m} \mathbf{x}_{m}^{\dagger}\right] \mathbf{H}_{m}^{\dagger}+\mathbf{\Phi}_{m}\right)}{\operatorname{det} \mathbf{\Phi}_{m}}
$$

where

$$
\boldsymbol{\Phi}_{m}=\sum_{\substack{\tilde{m}=1 \\ \tilde{m} \neq m}}^{M} \mathbf{H}_{m} \mathrm{E}\left[\mathbf{x}_{\tilde{m}} \mathbf{x}_{\tilde{m}}^{\dagger}\right] \mathbf{H}_{m}^{\dagger}+\sigma_{n}^{2} \mathbf{I} .
$$

As a result, the overall system spectral efficiency of a multiuser MIMO system is given by

$$
\bar{C}=\frac{1}{N} \sum_{m=1}^{M} C_{m} \quad \text { bits/transmission. }
$$

The above capacity expression is generally not exact, but is a good approximation when the number of users or the number of transmit antennas is large by central limit theorem (i.e., $M>3$ or $n_{T}>3$ typically). Because this approximate capacity expression inherits the relations between the co-channel signals, capacity based on (63) is still used for primary performance comparison even for the case when the number of users and the number of transmit antennas are small.

\section{B. Results}

In Figs. 3 and 4, results are provided for a three-user $(M=3)$, six BS antennas $\left(n_{T}=6\right)$, three antennas per MS $\left(n_{R, 1}=n_{R, 2}=n_{R, 3}=3\right)$, and two spatial dimensions per MS ( $K_{1}=K_{2}=K_{3}=2$ ) MIMO system. This configuration satisfies the minimum requirement of the numbers of antennas. Although the conditions we show are not sufficient and cannot guarantee the existence of joint multiuser channel diagonalization, simulation results will reveal that the diagonalization is achieved under all configurations we have investigated, whenever the "necessary" conditions are satisfied. The performance of JMMS significantly outperforms other approaches and more than 100 times reduction in average user BER is possible for JMMS when compared with MTXSINR. Similar comparisons can be made in Fig. 4. Results illustrate that JMMS achieves significant performance improvement compared with other schemes. Also note that the capacity of JMMS follows the same trend as that of the performance bound (which assumes no $\mathrm{CCI}$ ). The capacity performance of JMMS is just a 4-dB shift of that of the bound. Therefore, JMMS effectively eliminates all the CCI while keeping the diversity advantages of the system to achieve high capacity. Additionally, results demonstrate that the multiuser adaptation by ISBM does not work effectively, so their performance is not as good as that of MTXSINR.

To further enhance the system performance, more receive antennas can be used for gaining additional space diversity, if possible. In Figs. 5 and 6, results are provided for the same configurations as that in the last two figures, except that the number of antennas for each MS is equal to the total number of co-channel signals of the system (i.e., $n_{R, m}=K_{1}+K_{2}+K_{3}$ for $m=1,2,3)$. A close observation from Fig. 5 indicates that MTXSINR is much better than JADE, DTx, and SVD-MMSE. But ISBM has even better performance compared with MTXSINR. However, much better average user BER can be achieved by JMMS. It is noted that the diversity advantage (slope) of JMMS is also the best compared with the other schemes. Specifically, average user bit-error probability as low as $10^{-4}$ can be achieved with an average SNR less than $3 \mathrm{~dB}$ in a three-user system. Moreover, results in Fig. 4 show that among all the schemes, JMMS is the best in terms of maximizing the overall system capacity and its performance follows the same trend as the loose upper bound. In contrast to the previous results, the performance of ISBM is now close to or slightly inferior than that of JMMS. (Similar computer simulations have concluded that ISBM is only effective for the case when the number of receive antennas for each MS is greater than or equal to the total number of co-channel signals within the system.) In addition, results demonstrate that under this configuration, the performance of MTXSINR degrades and the performance difference between JMMS and MTXSINR is remarkable. This can be explained by the fact 


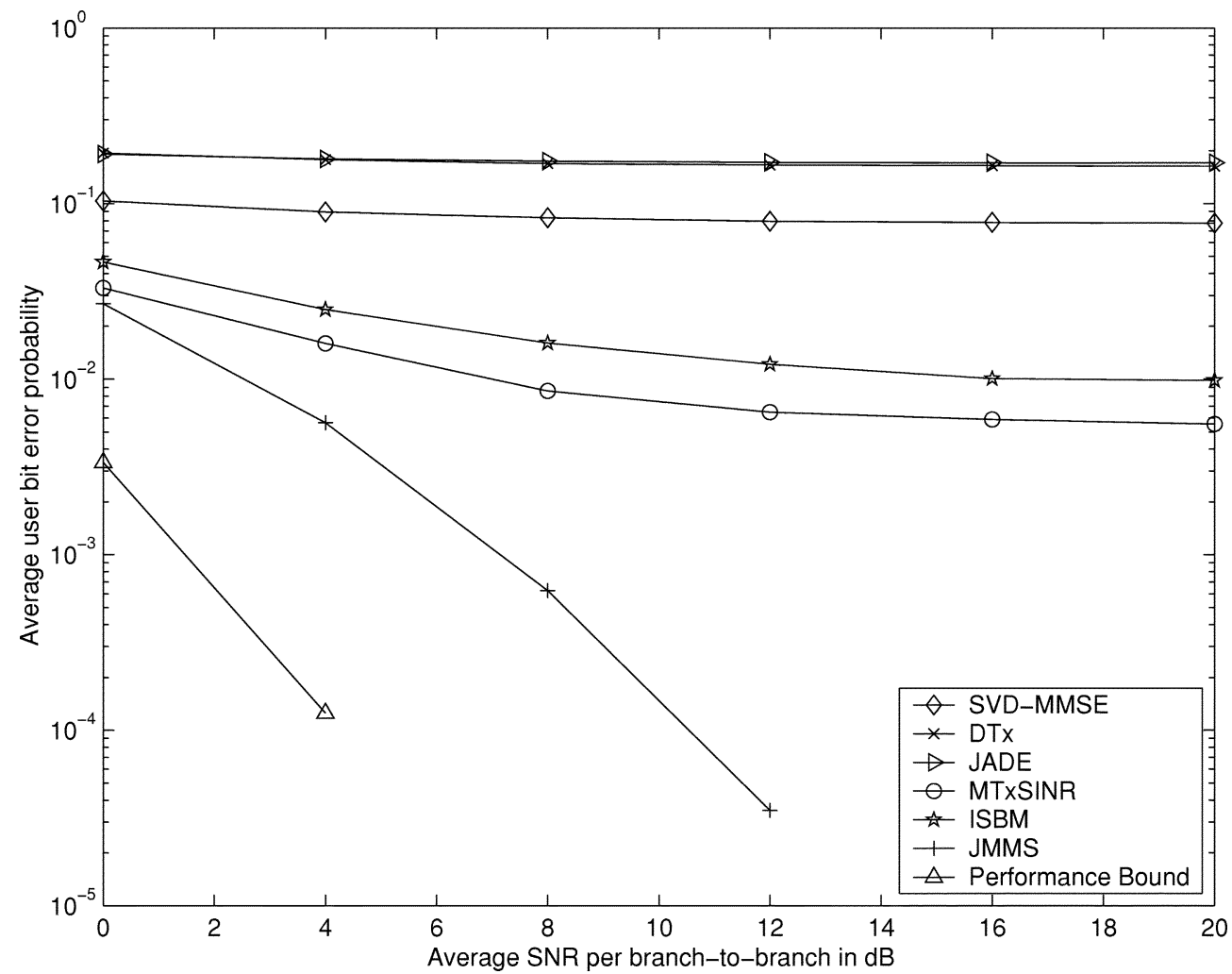

Fig. 3. Average user bit-error probability versus SNR for three-user MIMO systems. Six BS antennas, three antennas per MS, and $\left(K_{1}, K_{2}, K_{3}\right)=(2,2,2)$.

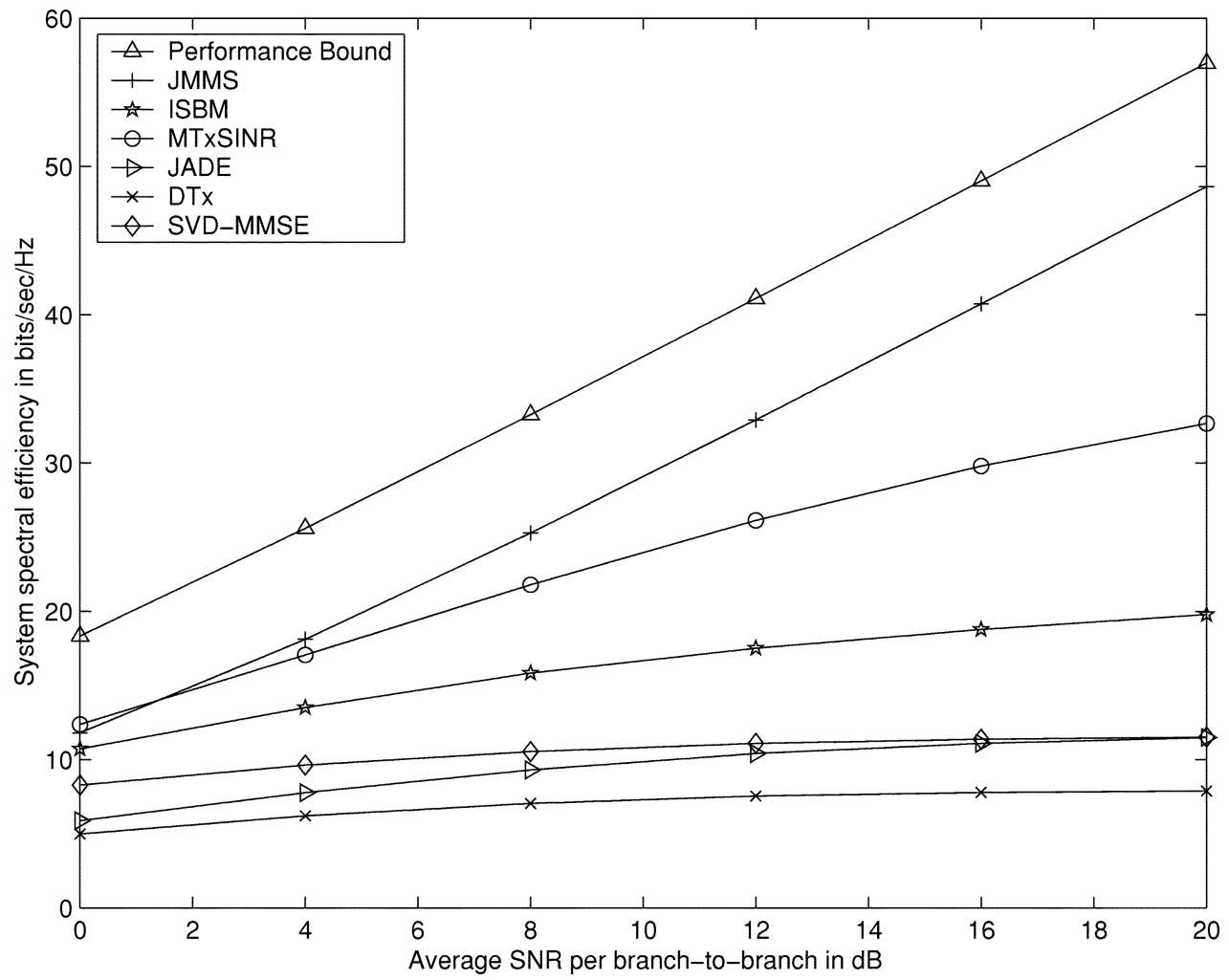

Fig. 4. Capacity versus SNR for three-user MIMO systems. Six BS antennas, three antennas per MS, and $\left(K_{1}, K_{2}, K_{3}\right)=(2,2,2)$

that the solution of MTxSINR comes from the maximization of the lower bound of the system spectral efficiency and as the number of users increases, the lower bound is inaccurate and, hence, the MTxSINR weights are unable to control all the CCI. The performance of DTx, JADE, and SVD-MMSE is not good, which implies that CCI minimization at the transmitter 


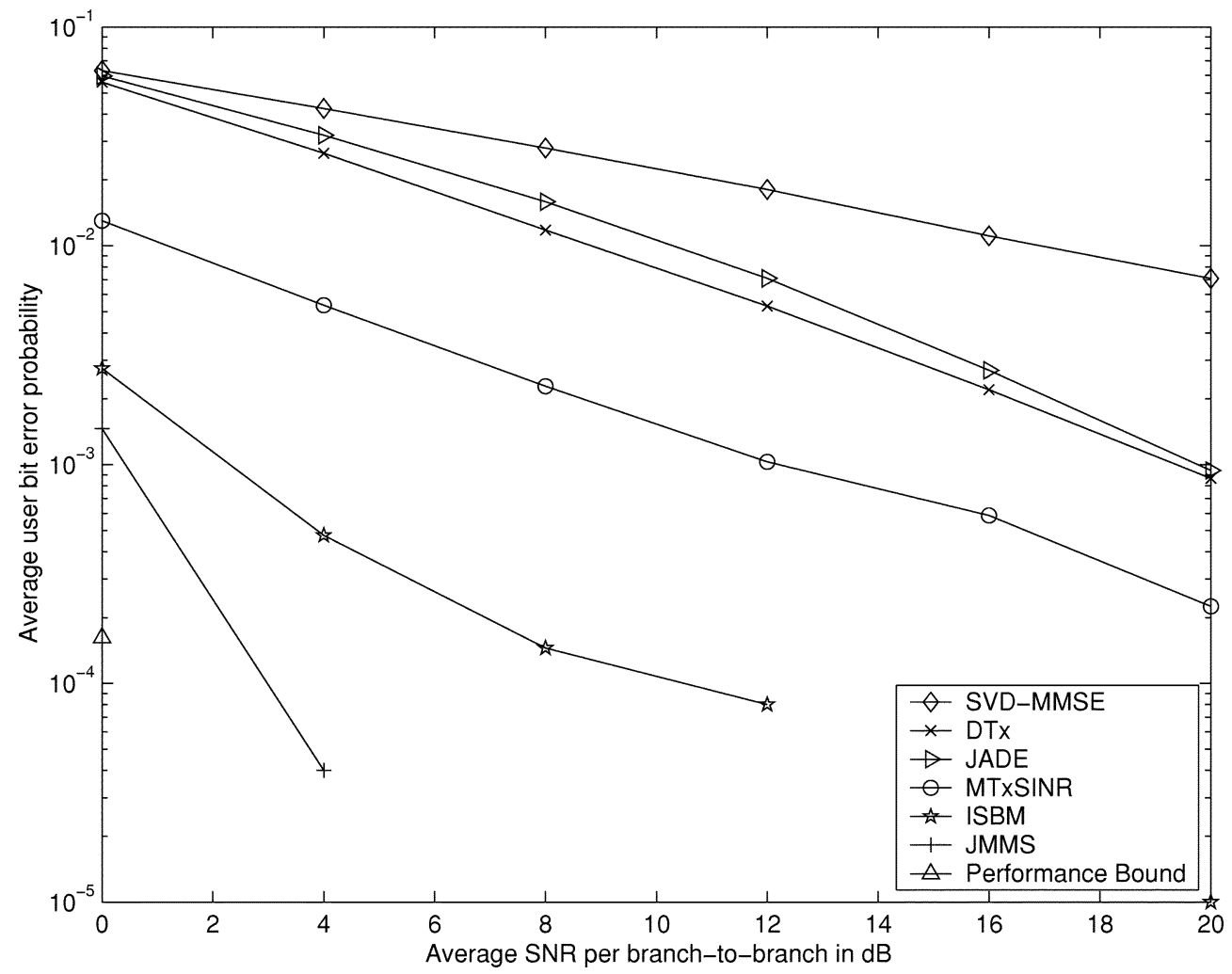

Fig. 5. Average user bit-error probability versus SNR for three-user MIMO systems. Six BS antennas, six antennas per MS, and $\left(K_{1}, K_{2}, K_{3}\right)=(2,2,2)$.

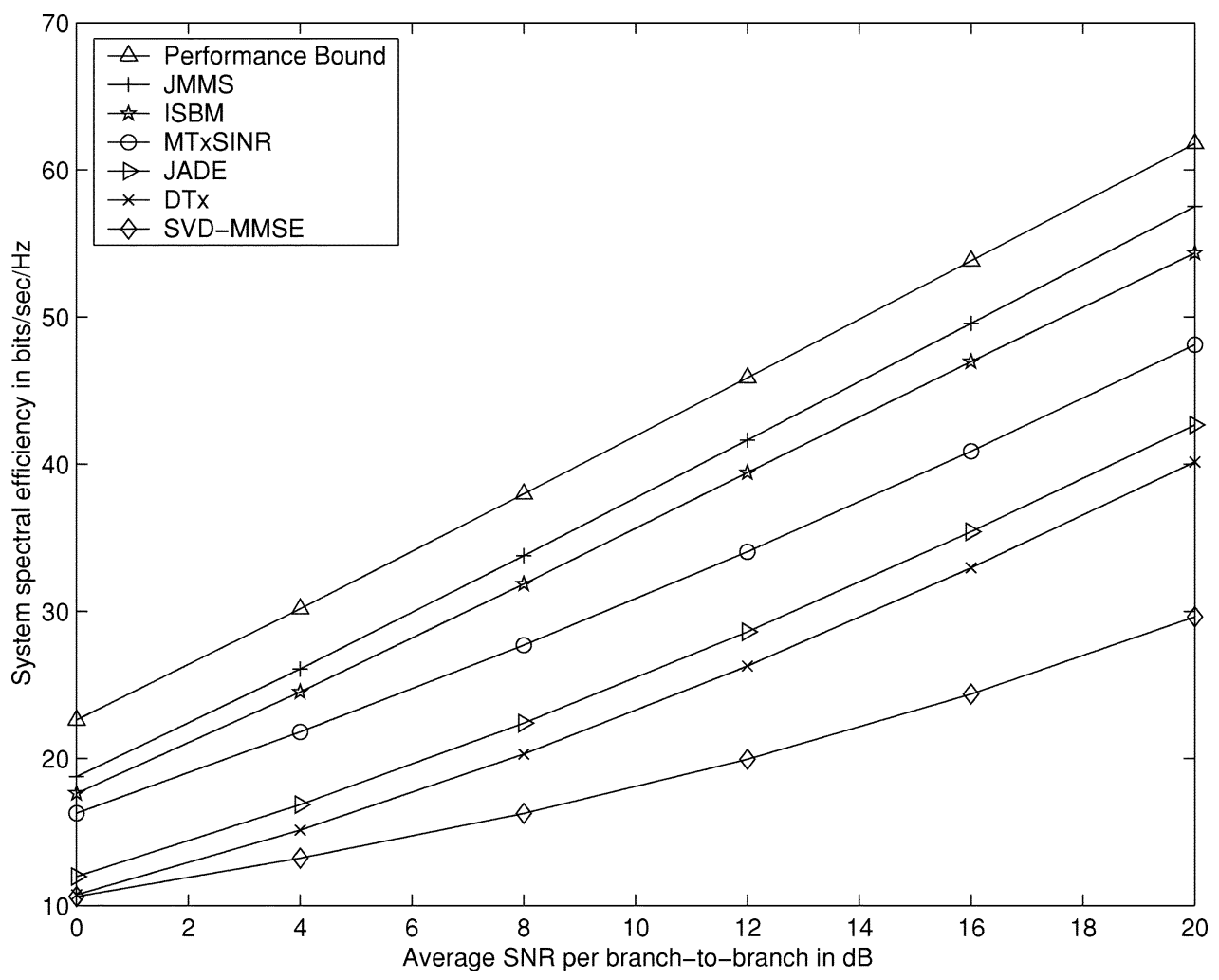

Fig. 6. Capacity versus SNR for three-user MIMO systems. Six BS antennas, six antennas per MS, and $\left(K_{1}, K_{2}, K_{3}\right)=(2,2,2)$.

side is important. One more thing worth mentioning is that by comparing the results in Fig. 6 with those in Fig. 4, we note that the performance of JMMS with three antennas per MS is even superior to that of MTxSINR with six antennas per MS for SNR $>17 \mathrm{~dB}$. This means that we could manage six co-channel signals with only three antennas per MS. Specifically, $48 \mathrm{~b} / \mathrm{s} / \mathrm{Hz}$ 


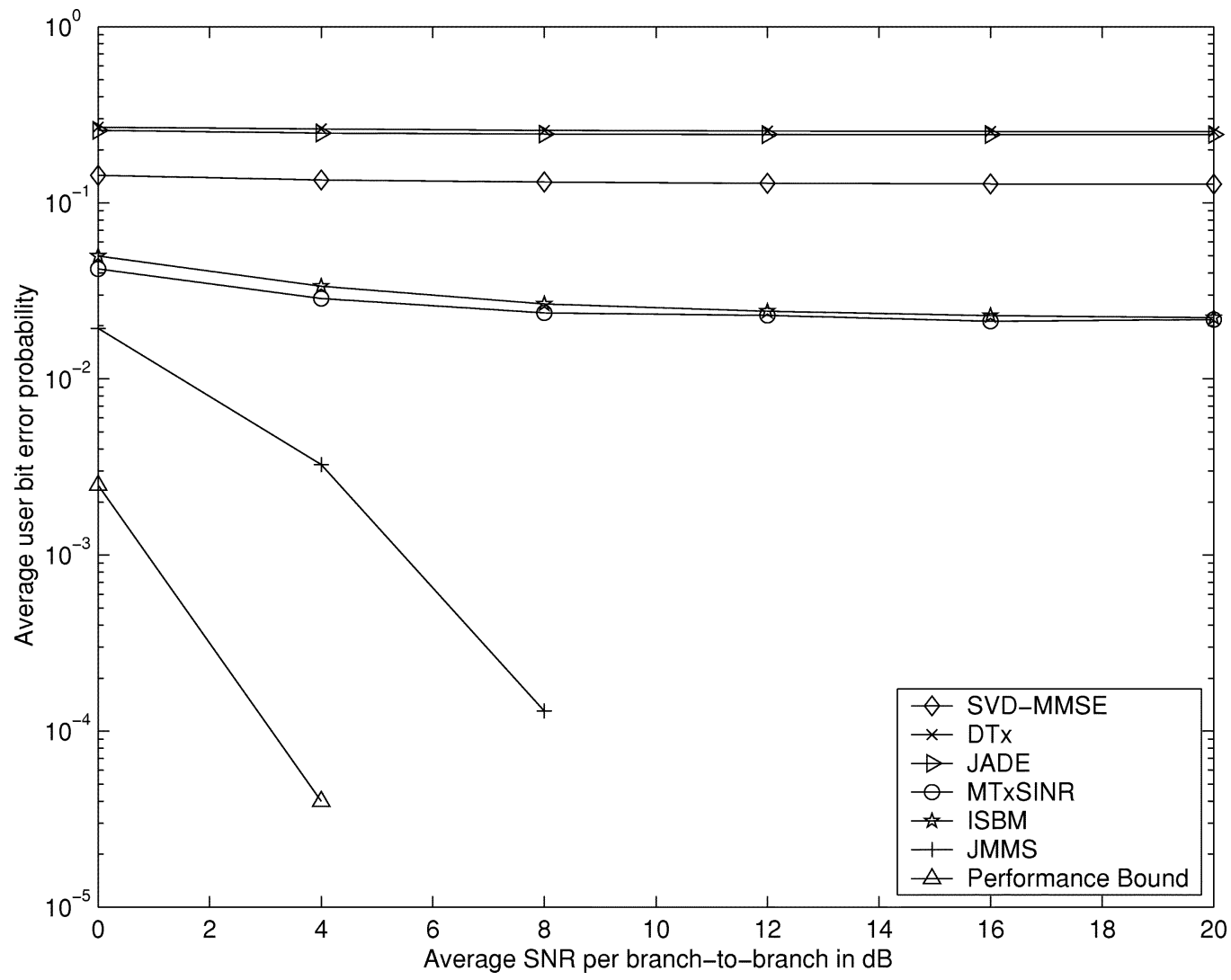

Fig. 7. Average user bit-error probability versus SNR for six-user MIMO systems. Six BS antennas, two antennas per MS, and $\left(K_{1}, K_{2}, K_{3}, K_{4}, K_{5}, K_{6}\right)=$ $(1,1,1,1,1,1)$.

(16 b/s/Hz per MS on average) can be achieved at an average $\mathrm{SNR}=20 \mathrm{~dB}$.

We conclude this section by providing results of the system where the number of co-channel users is large and each MS could only be allowed to have a small number of antennas. Results in Figs. 7 and 8 are provided for a six-user $(M=6)$ MIMO system with six BS antennas $\left(n_{T}=6\right)$, two antennas per MS $\left(n_{R, 1}=\cdots=n_{R, 6}=2\right)$, and one spatial dimension per MS $\left(K_{1}=K_{2}=\cdots=K_{6}=1\right)$. Notice that the total number of co-channel signals is strictly greater than the number of antennas per MS. Thus, interference cancellation relies greatly on the adaptation of BS antenna weights. A close observation of this figure indicates that JMMS achieves similar performance as that of the upper bound with only 3- to 4- $\mathrm{dB}$ degradation in average SNR. Because the bound is loose, especially for a large number of co-channel signals in the system, it is believed that JMMS is a promising tool for achieving the optimal multiuser separation in space. Results from Fig. 7 indicate that even for the case of a large number of users, JMMS works well as long as the necessary conditions are satisfied. Remarkably, the achievable diversity advantage of JMMS is nearly the same as that of the performance bound, and an average user BER of $10^{-4}$ is possible when the average SNR $>8 \mathrm{~dB}$. Consistent results for system capacity can be seen in Fig. 8. Similar to all the cases when the necessary conditions for JCD are satisfied, the overall system capacity can grow linearly as the average SNR increases and with the increasing rate as large as that of the performance bound. In particular, about $50 \mathrm{~b} / \mathrm{s} / \mathrm{Hz}$ (or $8.3 \mathrm{~b} / \mathrm{s} / \mathrm{Hz}$ per $\mathrm{MS}$ on average) can be obtained for SNR $>20 \mathrm{~dB}$ when using JMMS. It is also noted that the performances of ISBM and MTxSINR are degraded compared with the configuration with six antennas per MS. One more interesting remark is that with six transmit antennas at BS and two or three antennas at MS, the combined channel is more or less nonfading.

\section{Discussion}

In this section, we summarize some characteristics of our proposed system that we have observed. They are given as follows.

- Number of Transmit (BS) Antennas: The number of antennas at the transmitter (or BS) should be at least the total number of co-channel signals within the system for JCD to be achieved. However, to take full advantage of the algorithm, the number of transmit antennas should be exactly equal to the number of co-channel signals in the system so that the size of the transmit weight matrix $\mathbf{T}$ agrees with that of the updating matrix $\mathbf{Q}^{(u)}$ (i.e., $\left.n_{T}=K_{1}+K_{2}+\cdots+K_{M}\right)$.

- Number of Receive (MS) Antennas and the Number of Spatial Dimensions: The number of receive antennas at the $m$ th MS should be greater than the number of spatial dimensions of that mobile (i.e., $n_{R, m}>K_{m}$ ). For example, for a three-user system where User 1 transmits two data streams $\left(K_{1}=2\right)$, User 2 transmits three data streams $\left(K_{2}=3\right)$, and User 3 transmits five data streams $\left(K_{3}=5\right)$, the minimum number of antennas required for 


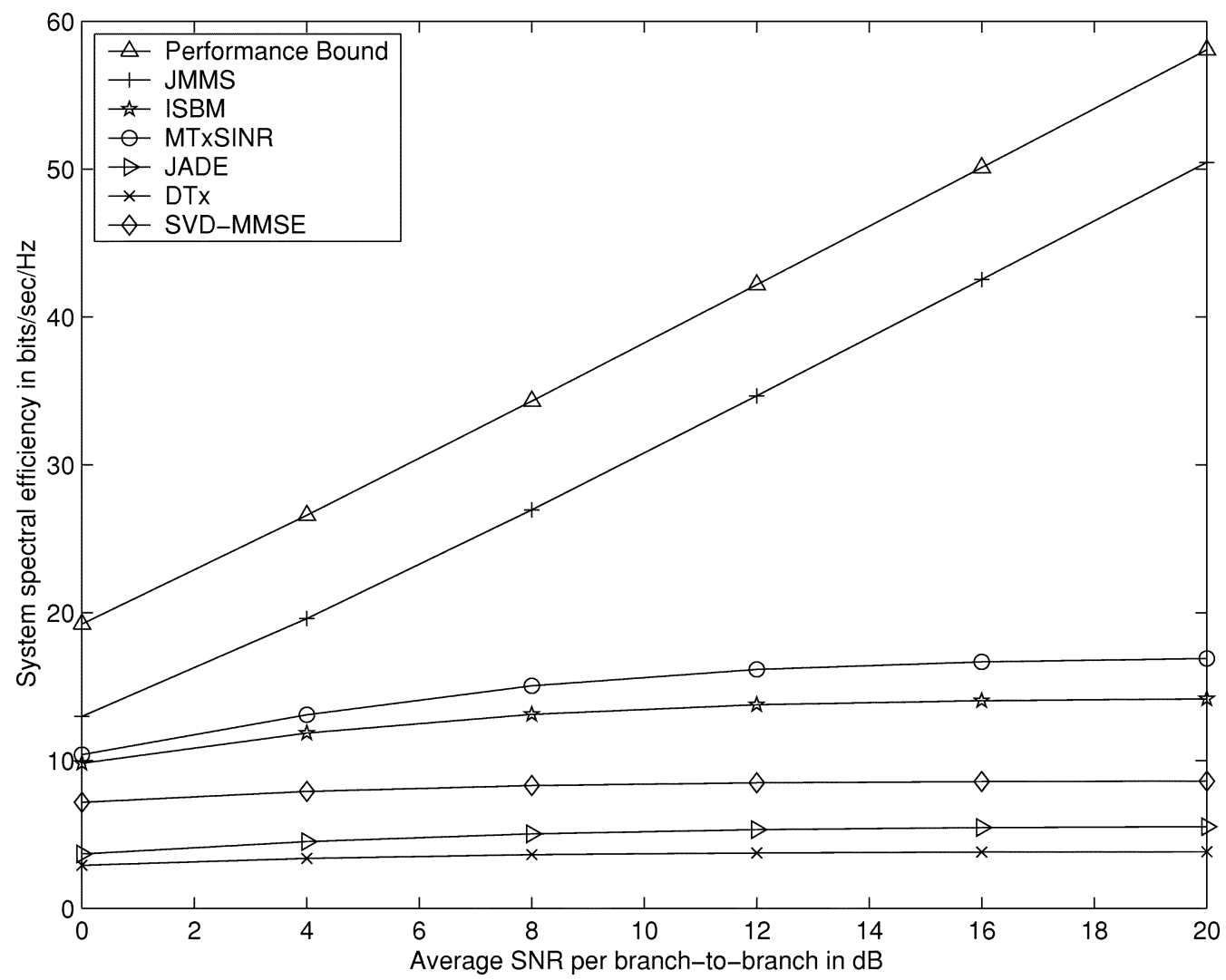

Fig. 8. Capacity versus SNR for six-user MIMO systems. Six BS antennas, two antennas per MS, and $\left(K_{1}, K_{2}, K_{3}, K_{4}, K_{5}, K_{6}\right)=(1,1,1,1,1,1)$.

the mobiles are three, four, and six for users 1,2 , and 3 , respectively. Following also Property 1, the minimum required number of BS antennas is 10 .

- Orthogonality of Multiuser MIMO Channels: Multichannel diagonalization obtained by JMMS decomposes an $M$-user MIMO downlink channel into $M$ uncoupled MIMO channels. Essentially, it becomes $M$ single-user systems with no interference (see Fig. 2). In addition, the parallel data streams (transmitted in spatial dimensions) for a particular user will be orthogonal with each other. This property allows the use of advanced techniques such as adaptive modulation and power allocation to further improve the user performance without deteriorating other users' performance.

- Capacity Expression: In the virtue of the orthogonality, the multiuser system capacity is given by (15). The capacity is a linear monotonic-increasing function against average SNR under the conditions of the minimum numbers of BS and MS antennas. A general rule of thumb for single-stream systems (i.e., $K_{1}=K_{2}=\cdots=K_{M}=1$ ) is that with two antennas at all mobiles, deploying $M$ antennas at BS can support $M$ co-channel users simultaneously at the same frequency and time slot without any cross interference. One more user can be accommodated by the system by having one more antenna at the BS.

Properties 1 and 2 agree with the necessary conditions in Section III-C for JCD when the fading is flat. This suggests that the conditions can be considered to be sufficient for flat-fading channels though we are unable to provide the proof.

\section{CONCLUSION}

This paper describes a solution (JMMS) for the problem of performance enhancement in terms of both maximizing the system capacity and minimizing the average user bit-error probability of a multiuser MIMO system for wireless communications. In particular, we provide some necessary conditions for the solution to exist. The formulation of our solution is general and applicable for both frequency-flat and selective-fading channels.

Simulation results reveal that the proposed JMMS system significantly outperforms all the existing systems in any configuration when the conditions presented in Section III-C are satisfied. It has also been demonstrated that the multiuser capacity can grow linearly as SNR increases, similar to the single user case, and this only occurs in JMMS. We have noted that JMMS can preserve the highest diversity advantage available in the system (achieves similar diversity order as without CCI). As a result, JMMS can be thought of as a generalization of SDM, and it is a promising technique for implementing multiuser SDM for high-rate and reliable wireless communications.

Our results provided in this paper assume perfect channel knowledge at both the transmitter and all receivers. In practice, CSI is estimated from the signals received in the uplink, and errors in channel estimation may be imposed that would certainly degrade the system performance. Further study is needed to quantify the robustness of the proposed system in the presence of channel estimation errors. Though conditions for JCD to exist have been provided as rules of thumb at the end of the paper, another interesting direction of research is to determine, 
analytically, the exact relation between the number of antennas, the number of users, the number of subchannels accommodated by each user, and the number of paths.

\section{REFERENCES}

[1] J. H. Winters, "Signal acquisition and tracking with adaptive arrays in the digital mobile radio system IS-54 with flat fading," IEEE Trans. Veh. Technol., vol. 42, pp. 377-384, Nov. 1993.

[2] J. Fuhl and A. F. Molisch, "Capacity enhancement and BER in a combined SDMA/TDMA system," in Proc. IEEE VTC, New York, Apr. 1996, pp. 1481-1485.

[3] G. G. Raleigh and J. M. Cioffi, "Spatio-temporal coding for wireless communications," IEEE Trans. Commun., vol. 46, pp. 357-366, Mar. 1998.

[4] J. B. Andersen, "Array gain and capacity for known random channels with multiple element arrays at both ends," IEEE J. Select. Areas Commun., vol. 18, pp. 2172-2178, Nov. 2000.

[5] K. K. Wong, R. D. Murch, and K. B. Letaief, "Optimizing time and space MIMO antenna system for frequency selective fading channels," IEEE J. Select. Areas Commun., vol. 19, pp. 1395-1407, July 2001.

[6] R. Stridh, M. Bengtsson, and B. Ottersten, "System evaluation of optimal downlink beamforming in wireless communication," in Proc. IEEE VTC Fall 2001, vol. 1, 2001, pp. 343-347.

[7] K. K. Wong, R. S.-K. Cheng, K. B. Letaief, and R. D. Murch, "Adaptive antennas at the mobile and base stations in an OFDM/TDMA system," IEEE Trans. Commun., vol. 49, pp. 195-206, Jan. 2001.

[8] K. K. Wong, R. D. Murch, and K. B. Letaief, "Performance enhancement of multiuser MIMO wireless communication systems," IEEE Trans. Commun., vol. 50, pp. 1960-1970, Dec. 2002.

[9] S. J. Grant and J. K. Cavers, "Multi-user channel estimation for detection of co-channel signals," IEEE Trans. Commun., vol. 49, pp. 1845-1855, Oct. 2001.

[10] Y. H. You, C. H. Park, M. C. Ju, J. H. Paik, K. W. Kwon, and J. W. Cho, "Channel estimation method with interference reduction for high-rate personal area networks," Electron. Lett., vol. 37, pp. 588-590, Apr. 2001.

[11] C. S. Yeh and Y. Lin, "Channel estimation using pilot tones in OFDM systems," IEEE Trans. Broadcast., vol. 45, pp. 400-409, Dec. 1999.

[12] T. M. Cover and J. A. Thomas, Elements of Information Theory. New York: Wiley, 1991.

[13] H. Lütkepohl, Handbook of Matrices. New York: Wiley, 1996.

[14] G. H. Golub and C. F. Van Loan, Matrix Computation, 3rd ed. Baltimore, MD: The Johns Hopkins Univ. Press, 1996.

[15] M. S. Pinsker, Information and Information Stability of Random Processes. San Francisco, CA: Holden Bay, 1964.

[16] G. J. Foschini and M. J. Gans, "On limits of wireless communications in a fading environment when using multiple antennas," in Wireless Personal Communications. Norwell, MA: Kluwer, 1998, ch. 6, pp. 311-335.

[17] R. T. Compton, Jr., Adaptive Antennas: Concept and Performance. Englewood Cliffs, NJ: Prentice-Hall, 1988.

[18] J. F. Cardoso and A. Souloumiac, "Jacobi angles for simultaneous diagonalization," SIAM J. Matrix Anal. Appl., vol. 17, no. 1, pp. 161-164, Jan. 1996.

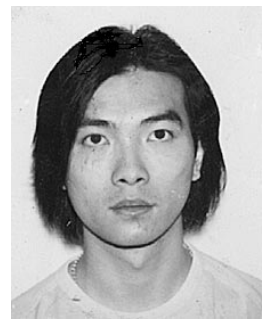

Kai-Kit Wong (S'98-M'01) received the B.Eng., M.Phil., and Ph.D. degrees in electrical and electronic engineering from The Hong Kong University of Science and Technology, Hong Kong, in 1996, 1998, and 2001, respectively.

Since October 2001, he has been with the Department of Electrical and Electronic Engineering, The University of Hong Kong, where he is a Research Assistant Professor. He has worked in several areas including smart antennas, space-time coding, and equalization. His current research interests center around the joint optimization of smart antennas for multiuser wireless communications systems.

Dr. Wong was a co-recipient of the IEEE Vehicular Technology Society (VTS) Japan Chapter Award of the VTC2000-Spring, Japan.

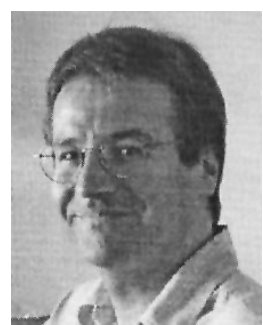

Ross D. Murch (S'85-M'87-SM'98) received the Bachelor's degree in electrical and electronic engineering (with first class honors) and the Ph.D. degree in electrical and electronic engineering from the University of Canterbury, Christchurch, New Zealand, in 1986 and 1990, respectively.

$\mathrm{He}$ is an Associate Professor in the Department of Electrical and Electronic Engineering at The Hong Kong University of Science and Technology, Hong Kong. From 1990 to 1992, he was a Postdoctoral Fellow in the Department of Mathematics and Computer Science at Dundee University, Scotland. From 1992 to 1998, he was an Assistant Professor in the Department of Electrical and Electronic Engineering at The Hong Kong University of Science and Technology. His current research interests in wireless communications include MIMO antenna systems, smart antenna systems, compact antenna design, and short range communications. $\mathrm{He}$ is the holder of several U.S. patents related to wireless communications, has authored over 100 published papers, and acts as a consultant for the industry.

Prof. Murch has been on the Editorial Board of IEEE TRANSACTIONS ON WIRELESS COMMUNICATIONS (known as IEEE JOURNAL ON SELECTED AREAS IN COMMUNICATION: WiRelesS SERIES before 2001) since 1999, and acts as a reviewer for several journals. He was the Chair of the Advanced Wireless Communications Systems Symposium at ICC 2002, New York, and is also the founding Director of the Center for Wireless Information Technology at The Hong Kong University of Science and Technology which was begun in August 1997. From August to December 1998, he was on sabbatical leave at Allgon Mobile Communications (manufactured 1 million antennas per week), Sweden and AT\&T Research Laboratories, NJ. During his undergraduate studies, he was the recipient of several academic prizes including the John Blackett prize for engineering and also the Austral Standard Cables prize. During his Ph.D. studies, he was awarded an RGC and also a New Zealand Telecom scholarship. He is a Chartered Engineer. In 1996 and 2001, he won engineering teaching excellence appreciation awards.

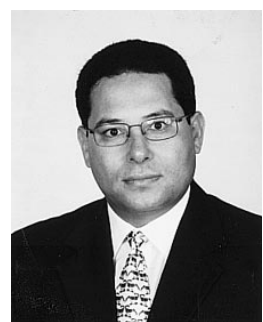

Khaled Ben Letaief (S'85-M'86-SM'97-F'03) received the B.S. degree (with distinction) and the M.S. and Ph.D. degrees from Purdue University, West Lafayette, IN, in 1984, 1986, and 1990, respectively, all in electrical engineering.

From January 1985 and as a Graduate Instructor in the School of Electrical Engineering at Purdue University, he has taught courses in communications and electronics. From 1990 to September 1993, he was a faculty member in the Department of Electrical and Electronic Engineering at the University of Melbourne, Australia, where he was also a Member of the Center for Sensor Signal and Information Systems. Since September 1993, he has been with the Department of Electrical and Electronic Engineering at The Hong Kong University of Science and Technology (HKUST), Hong Kong, where he is now a Professor and Head. His current research interests include wireless and mobile communications, OFDM, space-time processing for wireless systems, multiuser detection, wireless multimedia communications, and CDMA systems.

Dr. Letaief was appointed the founding Editor-in-Chief of the IEEE TRANSACTIONS ON WIRELESS COMMUNICATIONS, in January 2002. He has served on the Editorial Boards of other journals including the IEEE TRANSACTIONS ON COMMUNICATIONS, IEEE Communications Magazine, Wireless Personal Communications, and IEEE JouRnal ON SELECTED AREAS IN COMmunicAtions-Wireless Series (as Editor-in-Chief). He served as the Technical Program Chair of the 1998 IEEE Globecom Mini-Conference on Communications Theory, held in Sydney, Australia. He is also the Co-Chair of the 2001 IEEE ICC Communications Theory Symposium, held in Helsinki, Finland. He is currently serving as Vice-Chair of the IEEE Communications Society Technical Committee on Personal Communications. He is also currently the Vice chair of Meeting and Conference Committee of the IEEE COMSOC Asia Pacific Board. In addition to his active research activities, he has also been a dedicated teacher committed to excellence in teaching and scholarship. He received the Mangoon Teaching Award from Purdue University in 1990, the Teaching Excellence Appreciation Award by the School of Engineering at HKUST in Spring 1995, Fall 1996, Fall 1997, and Spring 1999, and the Michael G. Gale Medal for Distinguished Teaching (highest university-wide teaching award and only one recipient/year is honored for his/her contributions). 